\title{
SGK1 induces vascular smooth muscle cell calcification through NF-kB signaling
}

\author{
Jakob VoelkI, ${ }^{1,2}$ Trang T.D. Luong, ${ }^{1}$ Rashad Tuffaha, ${ }^{3}$ Katharina Musculus, ${ }^{3}$ Tilman Auer, ${ }^{3}$ Xiaoming Lian,,${ }^{4}$ Christoph Daniel, \\ Daniel Zickler, ${ }^{4}$ Beate Boehme, ${ }^{1}$ Michael Sacherer, ${ }^{6}$ Bernhard Metzler, ${ }^{7}$ Dietmar Kuhl, ${ }^{8}$ Maik Gollasch, ${ }^{4}$ Kerstin Amann, ${ }^{5}$ \\ Dominik N. Müller, ${ }^{2,9,10,11}$ Burkert Pieske, ${ }^{1,11,12}$ Florian Lang, ${ }^{3}$ and loana Alesutan ${ }^{1,2,11}$
}

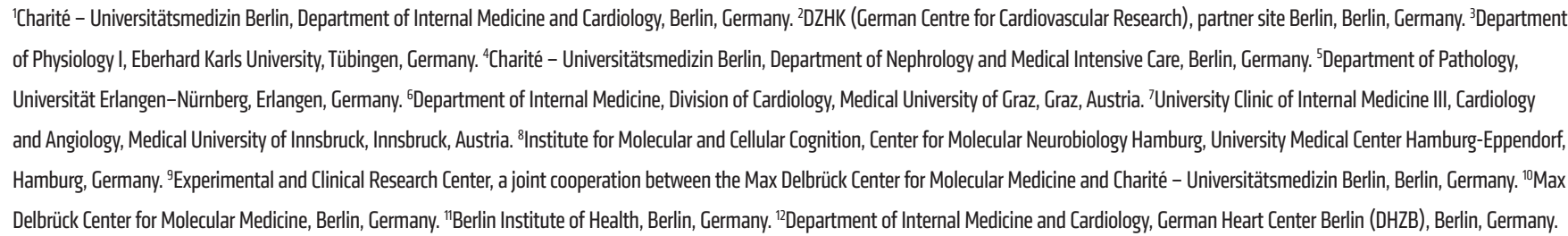

\begin{abstract}
Medial vascular calcification, associated with enhanced mortality in chronic kidney disease (CKD), is fostered by osteo-/chondrogenic transdifferentiation of vascular smooth muscle cells (VSMCs). Here, we describe that serum- and glucocorticoid-inducible kinase 1 (SCK1) was upregulated in VSMCs under calcifying conditions. In primary human aortic VSMCs, overexpression of constitutively active SGK1 $1^{5422 D}$, but not inactive SGK1 ${ }^{127 N}$, upregulated osteo-/ chondrogenic marker expression and activity, effects pointing to increased osteo-/chondrogenic transdifferentiation. SGK $1^{54220}$ induced nuclear translocation and increased transcriptional activity of NF-кB. Silencing or pharmacological inhibition of IKK abrogated the osteoinductive effects of SCK $1^{54220}$. Genetic deficiency, silencing, and pharmacological inhibition of SGK1 dissipated phosphate-induced calcification and osteo-/chondrogenic transdifferentiation of VSMCs. Aortic calcification, stiffness, and osteo-/chondrogenic transdifferentiation in mice following cholecalciferol overload were strongly reduced by genetic knockout or pharmacological inhibition of Sgk1 by EMD638683. Similarly, Sgk1 deficiency blunted vascular calcification in apolipoprotein E-deficient mice after subtotal nephrectomy. Treatment of human aortic smooth muscle cells with serum from uremic patients induced osteo-/chondrogenic transdifferentiation, effects ameliorated by EMD638683. These observations identified SGK1 as a key regulator of vascular calcification. SGK1 promoted vascular calcification, at least partly, via NF-KB activation. Inhibition of SGK1 may, thus, reduce the burden of vascular calcification in CKD.
\end{abstract}

\section{Introduction}

Medial vascular calcification is associated with cardiovascular events and mortality (1) of patients with chronic kidney disease (CKD) (2). In contrast to intimal atherosclerosis, vascular calcification is fostered by traditional and also by nontraditional cardiovascular risk factors, most importantly hyperphosphatemia (3). Hyperphosphatemia develops in patients with CKD as part of the mineral-bone disorder (4). Elevated phosphate levels promote the osteo-/chondrogenic transdifferentiation of vascular smooth muscle cells (VSMCs), which induces an active process of tissue mineralization (5). The osteo-/chondrogenic transdifferentiation of VSMCs is characterized by expression of osteo- and chondrogenic transcription factors, such as core-binding factor subunit $\alpha 1$ (CBFA1, also known as runt-related transcription factor 2

Authorship note: FL and IA contributed equally as co-senior authors. Conflict of interest: The authors have declared that no conflict of interest exists. Submitted: July 25, 2017; Accepted: April 17, 2018.

Reference information: J Clin Invest. 2018;128(7):3024-3040.

https://doi.org/10.1172/JCI96477.
[RUNX2]) (6) and msh homeobox 2 (MSX2) (7). Furthermore, the transformed VSMCs express tissue-nonspecific ALPL (alkaline phosphatase, liver/bone/kidney), which cleaves the powerful calcification inhibitor pyrophosphate (PPi) (8). The upregulation of alkaline phosphatase is, therefore, a critical event leading to mineral deposition in vascular calcification (8). Phosphate-induced remodeling of VSMCs is decisive for the mineralization of vascular tissue (9). However, the signaling cascades inducing the transdifferentiation of VSMCs during CKD remained ill defined.

In patients with $\mathrm{CKD}$, aldosterone plasma levels are enhanced (10). Aldosterone is a key factor inducing osteo-/chondrogenic transdifferentiation of VSMCs, and mineralocorticoid receptor antagonism reduces the extent of vascular calcification in animal models as well as the excessive cardiovascular mortality of hemodialysis patients (11-13). Also, cortisol levels are associated with mortality in end-stage renal disease, and glucocorticoids have been suggested to augment vascular calcification $(14,15)$. Both glucocorticoids and aldosterone exert their effects partly via serum- and glucocorticoid-inducible kinase 1 (SGK1) (16). SGK1 is known to exert deleterious effects of aldosterone 
A
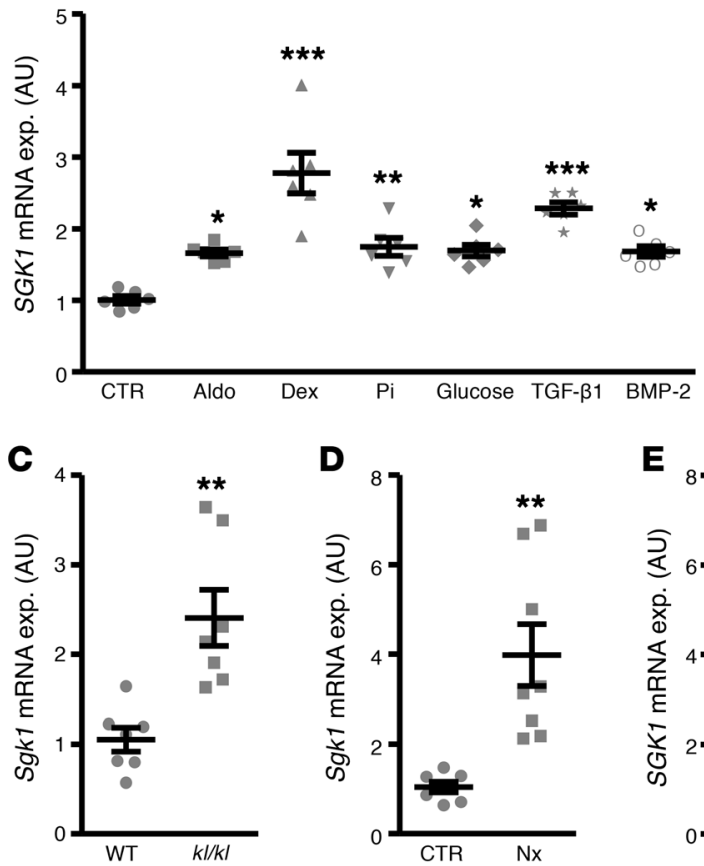

\section{D}
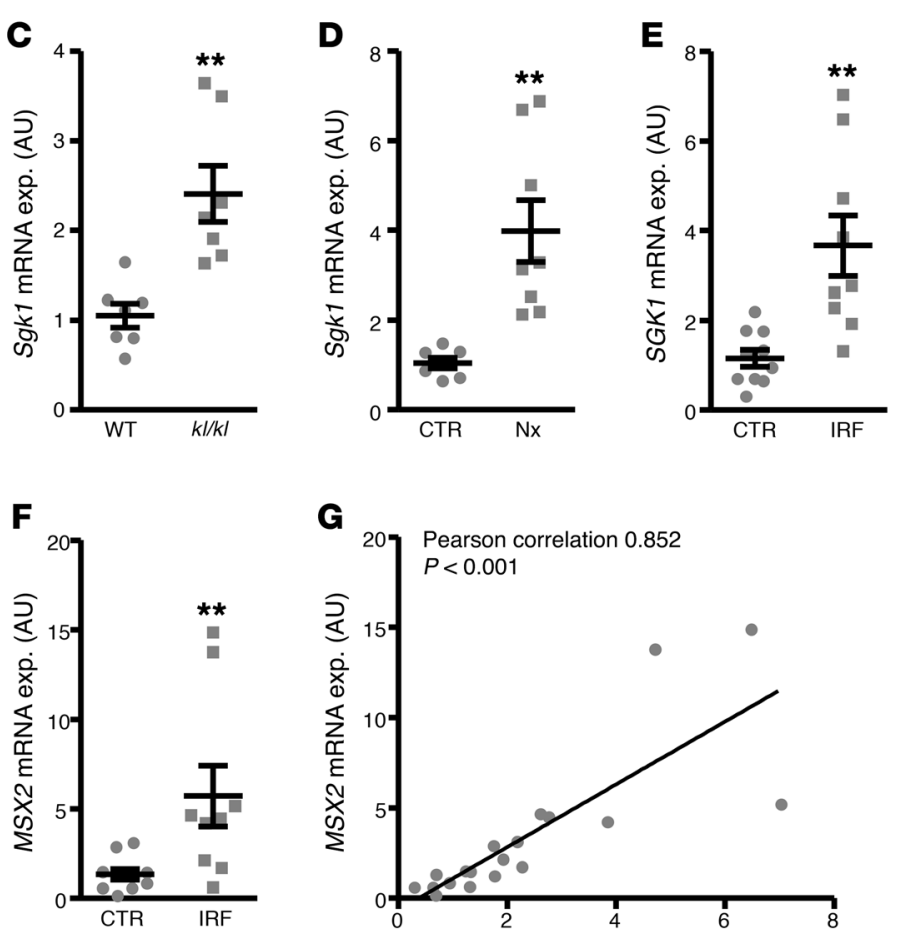

G

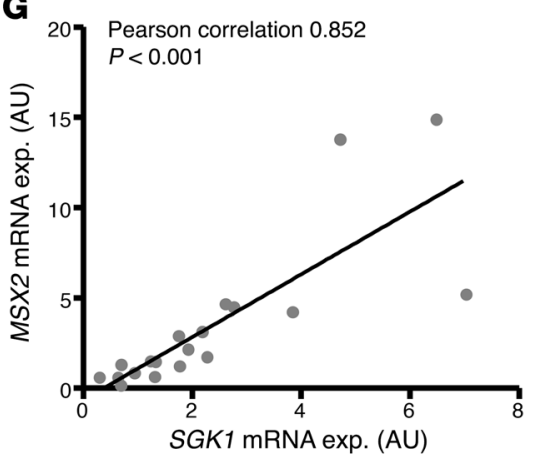

B
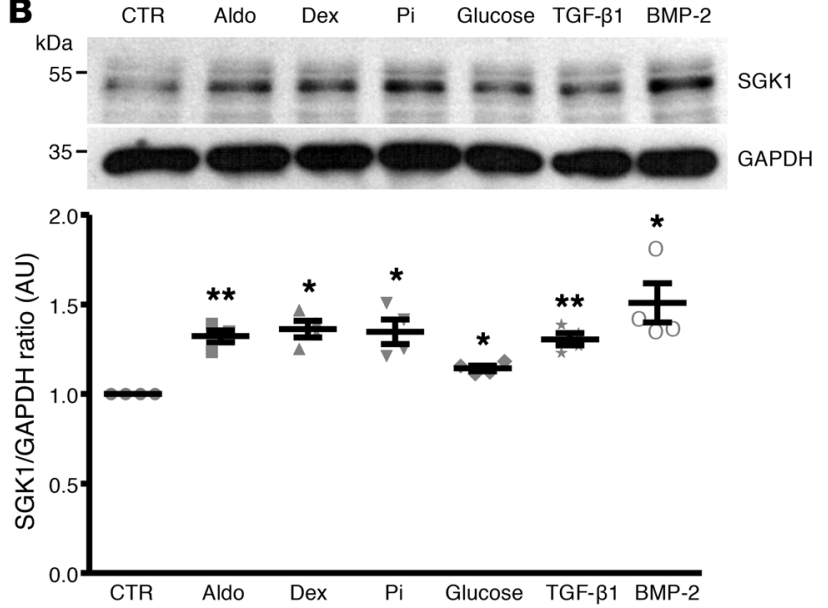

H
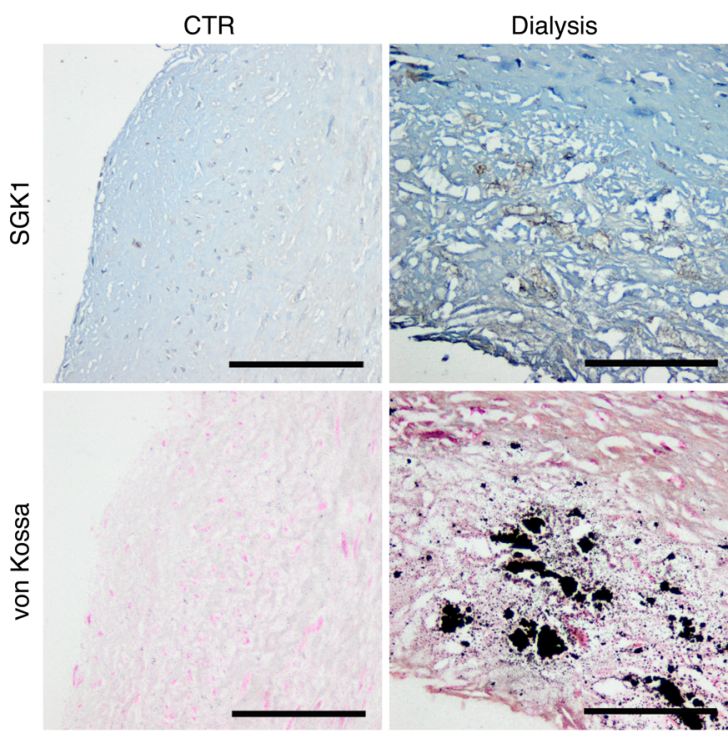

Figure 1. SGK1 expression in VSMCs. (A) Scatter dot plots and arithmetic means \pm SEM ( $n=6$ per group; arbitrary units [AU]) of SCK1 relative mRNA expression in primary HAoSMCs following treatment with control (CTR) or aldosterone (Aldo), dexamethasone (Dex), $\beta$-glycerophosphate (Pi), glucose, recombinant human TCF- $\beta 1$ protein, or recombinant human BMP-2 protein. (B) Representative original Western blots and scatter dot plots and arithmetic means \pm SEM ( $n=4$ per group; AU) of normalized SGK1/GAPDH protein ratio in HAoSMCs following treatment with triggers of osteo-chondrogenic transdifferentiation. ${ }^{*} P<0.05,{ }^{* *} P<0.01,{ }^{* *} P<0.001$ statistically significant vs. control-treated HAoSMCs (1-way ANOVA with Tukey-Honestly Significant Difference [HSD] [A] and with Games-Howell post hoc test [B]). (C and D) Scatter dot plots and arithmetic means \pm SEM (AU) of Sgk1 relative mRNA expression in aortic tissue from $\mathrm{kl} / \mathrm{kl}$ mice and WT mice (C, $n=7$ per group) and DBA mice without (CTR) or with subtotal nephrectomy (Nx) (D, $n=$ 7-8 per group). (E and $\mathbf{F})$ Scatter dot plots and arithmetic means \pm SEM (AU) of SGK1 (E) and MSX2 (F) relative mRNA expression in coronary artery tissue from patients with maintained (CTR, $n=10$ ) and impaired (IRF, $n=9$ ) renal function. ${ }^{* *} P<0.01$ statistically significant vs. WT mice, control-treated mice, or CTR patients, respectively (unpaired 2-tailed $t$ test for $\mathbf{C}-\mathbf{F}$ ). (G) Correlation of SCK1 and MSX2 relative mRNA expression (AU) in coronary artery tissue from CTR and IRF patients. $P$ represents the 2-tailed probability value of the Pearson correlation. (H) Representative original histological images $(n=5$ per group) showing SGK1 expression as well as ectopic calcification by von Kossa staining (calcification, gray to black) in coronary artery sections from control patients and dialysis patients. Scale bars: $100 \mu \mathrm{m}$.

and glucocorticoids in the cardiovascular system, such as hypertension, cardiac hypertrophy, cardiac fibrosis, and promotion of arrhythmias (17-21). SGK1 also contributes to vascular inflammation during atherosclerosis by regulating macrophage and VSMC migration $(22,23)$. After obstructive renal injury, SGK1 promotes renal fibrosis $(24,25)$. A specific inhibitor of SGK1 was already developed and successfully tested in vivo (26). SGK1 may contribute to progression of renal disease and was discussed as a possible therapeutic target (16).
SGK1 is involved in the regulation of various critical intracellular signaling pathways (16). SGK1 activates the nuclear factor $\kappa$-light chain enhancer of activated B cells (NF- $\mathrm{kB}$ ) by phosphorylating IKK $\alpha$ (27). IKK $\beta$ is required for SGK1-dependent activation of NF-KB (27). $\mathrm{NF}-\kappa \mathrm{B}$, in turn, has been described as an essential mediator of VSMC calcification, effects involving induction of tristetraprolin-mediated (TTP-mediated) destabilization of ankylosis protein homolog $($ ANKH) mRNA, a transmembrane protein involved in PPi transport of cells (28). Patients with CKD show an increased activation of 
A

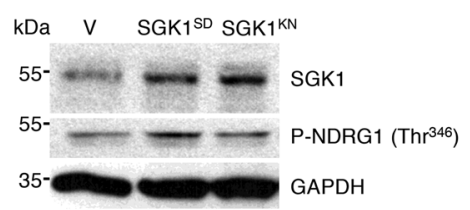

C

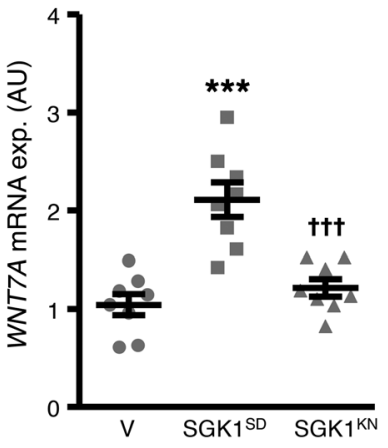

G

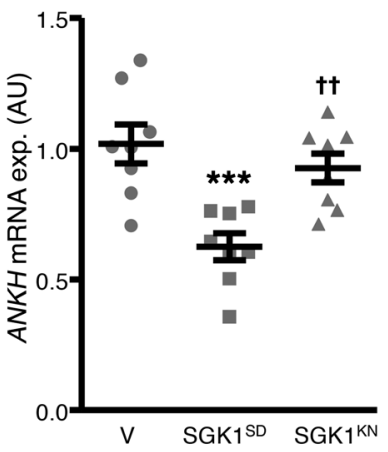

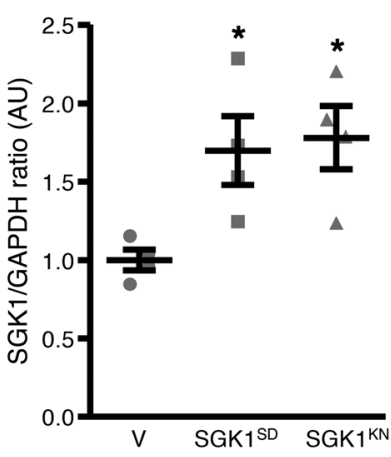
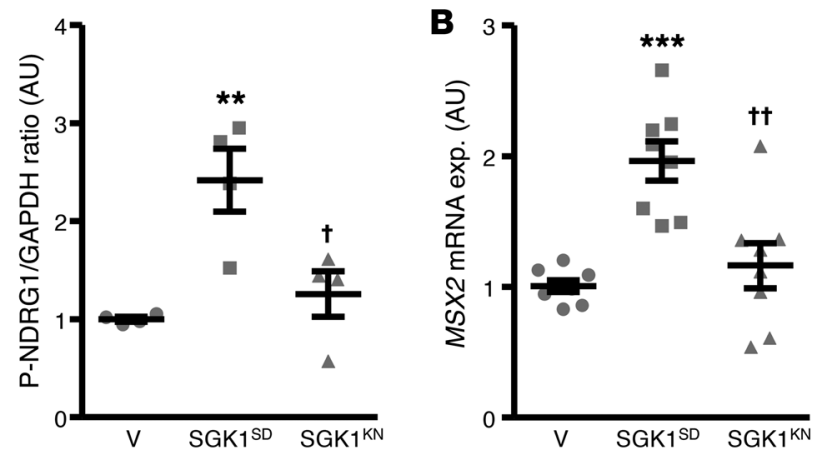

D

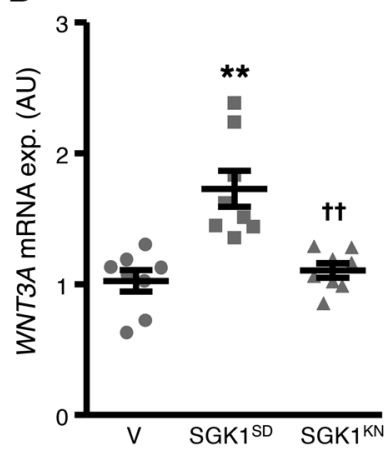

E

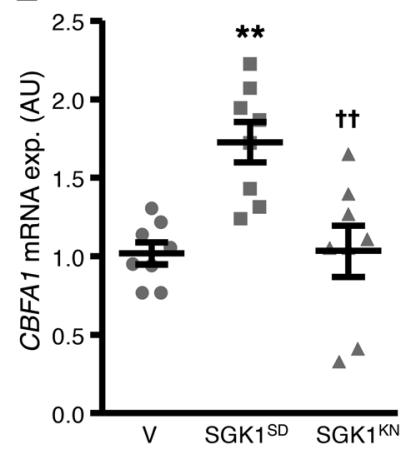

$\mathbf{F}$
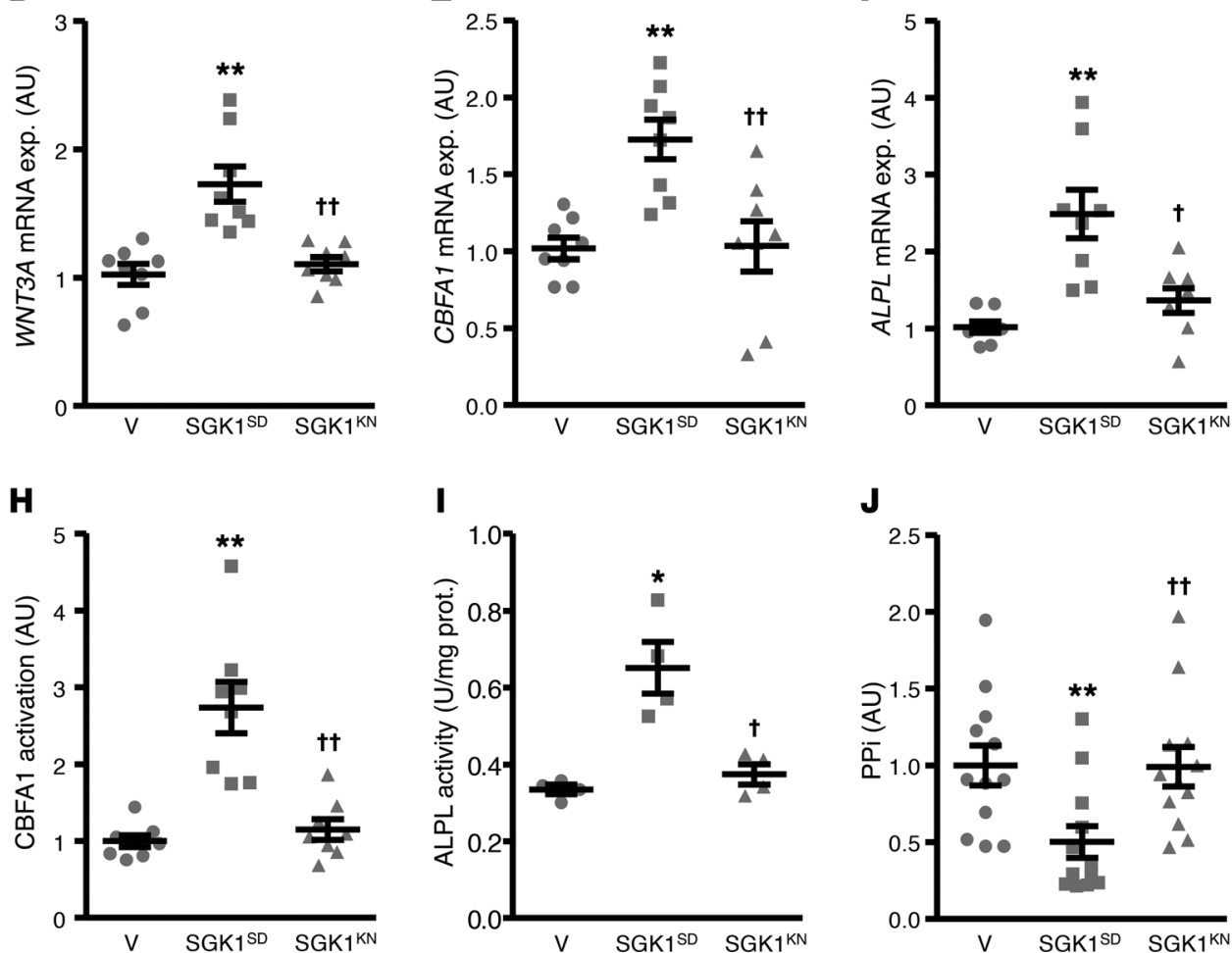

I

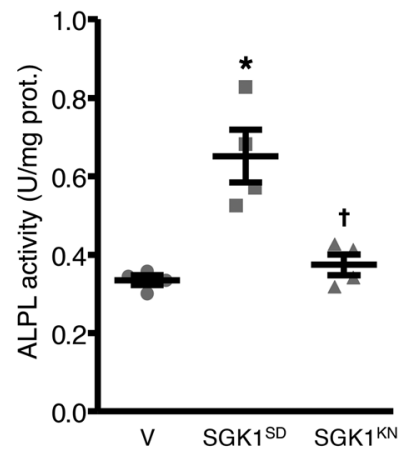

J

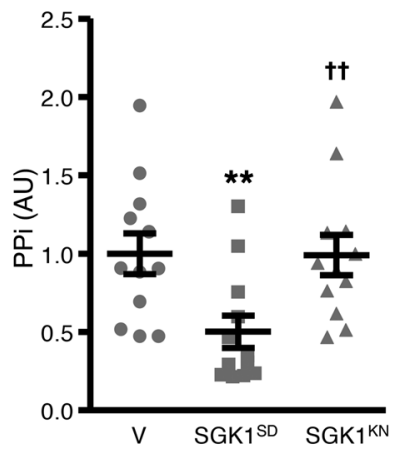

Figure 2. SGK1 overexpression induces osteo-/chondrogenic transdifferentiation of primary HAoSMCs. (A) Representative original Western blots and scatter dot plots and arithmetic means \pm SEM ( $n=4$ per group; AU) of normalized SCK1/GAPDH and phospho-NDRG1(Thr $\left.{ }^{346}\right) /$ GAPDH protein ratio in HAoSMCs following transfection with empty vector $(V)$, constitutively active SCK $1^{5422 D}$ (SGK1 $1^{\mathrm{SD}}$ ), or inactive SGK1 $1^{\mathrm{K} 127 \mathrm{~N}}$ (SGK1 ${ }^{\mathrm{KN}}$ ). (B-C) Scatter dot plots and arithmetic means \pm SEM ( $n=8$ per group; AU) of MSX2 (B), WNT7A (C), WNT3A (D), CBFA1 (E), ALPL (F), and ANKH (G) relative mRNA expression in HAoSMCs following transfection with empty vector $(\mathrm{V})$, constitutively active SGK1 $1^{\mathrm{S422D}}\left(\mathrm{SGK} 1^{\mathrm{SD}}\right)$, or inactive SGK1 ${ }^{\mathrm{K} 127 \mathrm{~N}}$ (SGK1 $1^{\mathrm{KN}}$ ). (H) Scatter dot plots and arithmetic means \pm SEM ( $n=8$ per group; AU) of normalized CBFA1 transcriptional activation in HAoSMCs following transfection with empty vector (V), constitutively active SCK $1^{5422 D}\left(\mathrm{SCK} 1^{\mathrm{SD}}\right)$, or inactive SCK1 ${ }^{\mathrm{K} 127 \mathrm{~N}}\left(\mathrm{SGK} 1^{\mathrm{KN}}\right)$. (I) Scatter dot plots and arithmetic means $\pm \mathrm{SEM}$ ( $n=4$ per group, U/mg protein) of alkaline phosphatase activity in HAoSMCs following transfection with empty vector $(\mathrm{V})$, constitutively active SGK $1^{\mathrm{S} 222 \mathrm{D}}$ (SGK1 $\left.1^{\mathrm{SD}}\right)$, or inactive SCK $1^{\mathrm{K} 127 \mathrm{~N}}\left(\mathrm{SGK} 1^{\mathrm{KN}}\right.$ ). (J) Scatter dot plots and arithmetic means \pm SEM ( $n=12$ per group; AU) of normalized PPi levels in the cell culture medium of HAoSMCs following transfection with empty vector $(\mathrm{V})$, constitutively active $\mathrm{SGK} 1^{\mathrm{S422D}}\left(\mathrm{SGK} 1^{\mathrm{SD}}\right)$, or inactive $\mathrm{SGK} 1^{\mathrm{K} 127 \mathrm{~N}}\left(\mathrm{SGK} 1^{\mathrm{KN}}\right)$. ${ }^{*} P<0.05,{ }^{* *} P<0.01$, ${ }^{* * *} P<0.001$ statistically significant vs. V-transfected HAOSMCs; ${ }^{\dagger} P<0.05,{ }^{\dagger+} P<0.01,{ }^{\dagger+\dagger} P<0.001$ statistically significant vs. SGK1 ${ }^{\text {SD }}$-transfected HAoSMCs (1-way ANOVA with Tukey-HSD post hoc test for $\mathbf{A}-\mathbf{C}, \mathbf{E}, \mathbf{G}$, and $\mathbf{J}$ and with Games-Howell post hoc test for $\mathbf{D}, \mathbf{F}, \mathbf{H}$, and I).

$\mathrm{NF}-\mathrm{kB}$ in the vasculature (28). In turn, increased expression of Sgk1 was observed in vascular tissue of rats after adenine-induced renal failure (12). At least in kidney biopsies, SGK1 expression is increased by advanced renal disease (29). Accordingly, SGK1 may be involved in osteo-/chondrogenic transdifferentiation of VSMCs and serve as a therapeutic target to limit the progression of vascular calcification. The current study, therefore, investigated whether SGK1 may contribute to vascular calcification during hyperphosphatemia.

\section{Results}

Regulation of vascular SGK1 expression during calcifying conditions. A first series of experiments explored the effect of known triggers of osteo-/chondrogenic transdifferentiation and calcification of VSMCs on vascular SGK1 expression. As illustrated in Figure 1, A and $\mathrm{B}$, treatment with aldosterone, dexamethasone, phosphate, glucose, recombinant human TGF- $\beta 1$ protein, or recombinant human BMP-2 protein significantly upregulated SGK1 mRNA 
and protein expression in primary human aortic smooth muscle cells (HAoSMCs). These effects were paralleled by increased mRNA expression of the osteogenic transcription factor MSX2 in all groups of HAoSMCs treated with triggers of VSMC calcification (Supplemental Figure 1; supplemental material available online with this article; https://doi.org/10.1172/JCI96477DS1). The mRNA expression of $S G K 1$ was significantly correlated with MSX2 mRNA expression in HAoSMCs (Pearson correlation 0.332, $P<0.05)$. Similarly, $S g k 1$ mRNA expression was significantly higher in aortic tissue of hyperphosphatemic klotho-hypomorphic mice $(k l / k l)$ as compared with WT mice (Figure 1C) and of mice with subtotal nephrectomy as compared with control-treated mice (Figure 1D), 2 mouse models of CKD-related vascular calcification.

The mRNA expression of SGK1 and of osteogenic marker $M S X 2$ was higher in human coronary arteries from patients with impaired renal function than in patients with normal renal function (Figure 1, E and F). More importantly, in both groups, SGK1 mRNA expression in human coronary arteries was significantly correlated with MSX2 mRNA expression (Figure 1G). Furthermore, as shown by histological analysis, SGK1 protein levels were higher in calcified human coronary artery sections from end-stage CKD patients on dialysis than from noncalcifying control patients and were localized in the calcified arterial regions (Figure $1 \mathrm{H}$ and Supplemental Table 1).

Effect of SGK1 overexpression on osteo-/chondrogenic transdifferentiation of primary HAoSMCs. To investigate whether SGK1 plays a role in vascular osteo-/chondrogenic induction, constitutively active SGK1 $1^{\mathrm{S} 422 \mathrm{D}}$ and inactive SGK1 $1^{\mathrm{K} 127 \mathrm{~N}}$ were overexpressed in HAoSMCs. As a result, overexpression of both SGK1 $1^{\text {S422D }}$ and SGK1 ${ }^{\mathrm{K} 127 \mathrm{~N}}$ significantly upregulated $S G K 1 \mathrm{mRNA}$ and protein expression to similarly elevated levels as compared with empty vector-transfected HAoSMCs (Figure 2A and Supplemental Figure 2A). Overexpression of SGK1 $1^{\mathrm{S} 22 \mathrm{D}}$, but not of SGK1 ${ }^{\mathrm{K} 127 \mathrm{~N}}$, significantly increased the phosphorylation of NDRG1, a known direct target of SGK1, pointing toward an increased SGK1 activity in SGK1 ${ }^{\text {S222D }}$-transfected HAoSMCs (Figure 2A).

In HAoSMCs, overexpression of $\mathrm{SGK}^{\mathrm{S422D}}$ significantly increased the MSX2/WNT signaling cascade as shown by upregulated MSX2, WNT7A, and WNT3A mRNA levels (Figure 2, B-D), osteogenic transcription factor $C B F A 1$ mRNA expression and transcriptional activity (Figure 2, E and $\mathrm{H}$ ), and osteogenic enzyme alkaline phosphatase (ALPL) mRNA expression and activity (Figure 2, F and I). Moreover, ANKH mRNA expression (Figure 2G) and PPi levels in the cell culture medium (Figure 2J) were significantly lower in SGK1 $1^{\text {S422D }}$-transfected HAoSMCs than in empty vector-transfected HAoSMCs. In contrast, overexpression of SGK1 ${ }^{\mathrm{K} 127 \mathrm{~N}}$ did not significantly affect the osteo-/chondrogenic signaling in HAoSMCs (Figure 2, B-J). These observations indicate that SGK1 induces osteo-/chondrogenic transdifferentiation of HAoSMCs, an effect requiring SGK1 kinase activity.

Role of NF- $\mathrm{KB}$ in SGK1-induced osteo-/chondrogenic transdifferentiation of primary HAoSMCs. To elucidate the underlying mechanisms of SGK1-dependent osteoinduction in HAoSMCs, the regulation of $\mathrm{NF}-\mathrm{\kappa B}$ transcription factor was investigated. In HAoSMCs, overexpression of SGK1 $1^{\mathrm{S} 422 \mathrm{D}}$, but not of SGK1 ${ }^{\mathrm{K} 127 \mathrm{~N}}$, induced the nuclear translocation of NF- $\kappa \mathrm{B}$ p 65 protein (Figure $3 \mathrm{~A}$ ), upregulated $\mathrm{NF}-\kappa \mathrm{B}-$ dependent transcriptional activity (Fig- ure 3B), and increased the mRNA expression of the RNA-destabilizing factor tristetraprolin (TTP, encoded by the ZFP36 gene), a downstream product of NF- $\kappa \mathrm{B}$ activation (Figure $3 \mathrm{C}$ ).

To further explore whether the effects of SGK1 overexpression on osteo-/chondrogenic signaling are dependent on $\mathrm{NF}-\mathrm{\kappa B}$ activation, HAoSMCs transfected with SGK1 ${ }^{\mathrm{S} 422 \mathrm{D}}$ (Supplemental Figure $2 \mathrm{~B}$ ) were additionally treated with inhibitors of the $\mathrm{NF}-\kappa \mathrm{B}$ pathway: BAY11-7082, an irreversible inhibitor of IKK $\alpha$ and phosphorylation of $\mathrm{I} \kappa \mathrm{B} \alpha$; parthenolide, which binds directly and inhibits IKK $\beta$; and BMS-345541, a highly selective inhibitor of IKK $\alpha$ and IKK $\beta$. The upregulation of MSX2, CBFA1, and ALPL mRNA expression induced by SGK1 ${ }^{\text {S422D }}$ overexpression in HAoSMCs was significantly blunted following additional treatment with each of the $3 \mathrm{NF}-\kappa \mathrm{B}$ pathway inhibitors (Figure 3, D-F). Furthermore, silencing of IKK $\alpha$ or IKK $\beta$ in HAoSMCs (Supplemental Figure 2, $\mathrm{C}-\mathrm{E}$ ) significantly blunted the upregulation of $M S X 2, C B F A 1$, and $A L P L$ mRNA expression in SGK1 ${ }^{\text {S422D }}$-transfected HAoSMCs without significantly affecting the expression of osteogenic markers in empty vector-transfected HAoSMCs (Figure 3, G-I). Thus, the procalcifying effects of SGK1 in HAoSMCs are, at least in part, dependent on NF- $\kappa \mathrm{B}$ activation.

Effect of Sgk1 deficiency on phosphate-induced osteo-/chondrogenic transdifferentiation of primary mouse aortic smooth muscle cells. To further explore the role of SGK1 in osteo-/chondrogenic transdifferentiation and calcification of VSMCs during hyperphosphatemic conditions, primary mouse aortic smooth muscle cells (MAoSMCs) were isolated from Sgk1-deficient mice $\left(\mathrm{sgk1} 1^{-/}\right)$or corresponding WT mice $\left(\mathrm{sgk}^{+/+}\right)$. Phosphate treatment upregulated $S g k 1$ mRNA expression in $\mathrm{sgk} 1^{+/+}$MAoSMCs (Figure 4A). As shown by confocal microscopy and analysis of fluorescence intensity, Sgk1 protein abundance was higher in phosphate-treated as compared with control-treated $\mathrm{sgk1}^{+/+}$ MAoSMCs, but no expression was observed in sgk1 $1^{-/-}$MAoSMCs (Figure 4B). Moreover, phosphate treatment induced nuclear

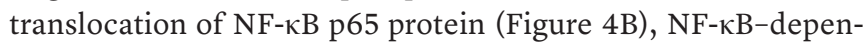
dent transcriptional activity (Figure $4 \mathrm{C}$ ), calcium deposition (Figure 4, D and E), alkaline phosphatase activity (Figure 4F), and $M s x 2, C b f a 1$, and $A l p l$ mRNA expression (Figure 4, G-I) in sgk1 ${ }^{+/+}$MAoSMCs, effects completely abrogated in sgk1 $1^{-/-}$MAoSMCs. No significant difference in smooth muscle cell-specific marker Tagln mRNA expression (encoding the $\mathrm{Sm} 22$ protein) was observed between control-treated $s g k 1^{+/+}$MAoSMCs and $\mathrm{sgk1}^{-/-}$MAoSMCs (Supplemental Figure 3). Phosphate treatment downregulated Tagln mRNA expression in sgk1 ${ }^{+/+}$MAoSMCs, but not in sgk1 $1^{-/-}$MAoSMCs.

Effect of SGK1 inhibition on phosphate-induced osteo-/chondrogenic transdifferentiation of primary HAoSMCs. Additional experiments were conducted to define the role of SGK1 as a possible therapeutic target in osteo-/chondrogenic transdifferentiation and calcification of HAoSMCs during hyperphosphatemic conditions. To this end, HAoSMCs were treated with phosphate without or with additional treatment with the highly selective SGK1 inhibitor EMD638683. As shown in Figure 5A, phosphate treatment significantly upregulated SGK1 mRNA expression in HAoSMCs, an effect not significantly modified by the presence of EMD638683. The SGK1 inhibitor EMD638683 did not alter the cytoplasmic local- 
A
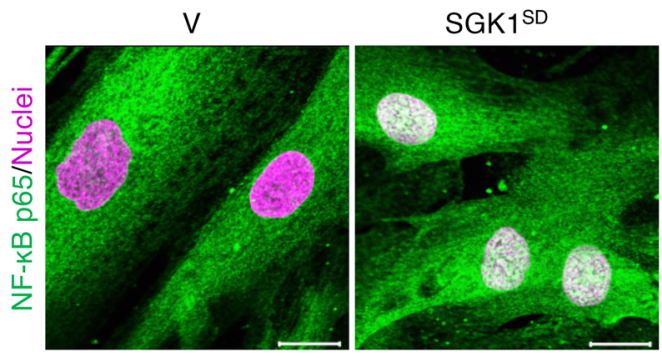

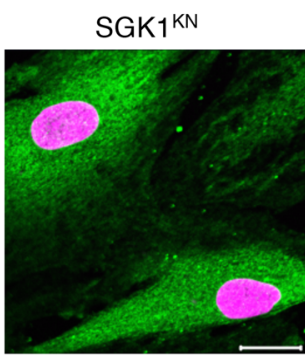

B

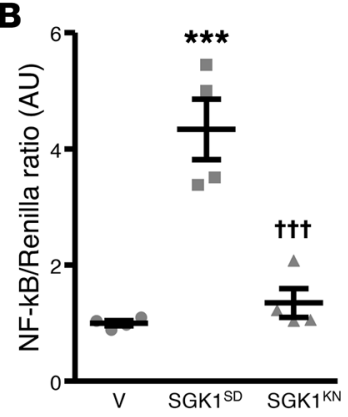

C

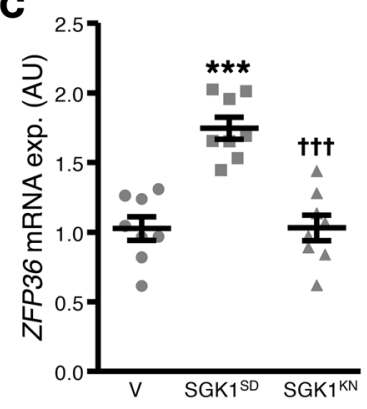

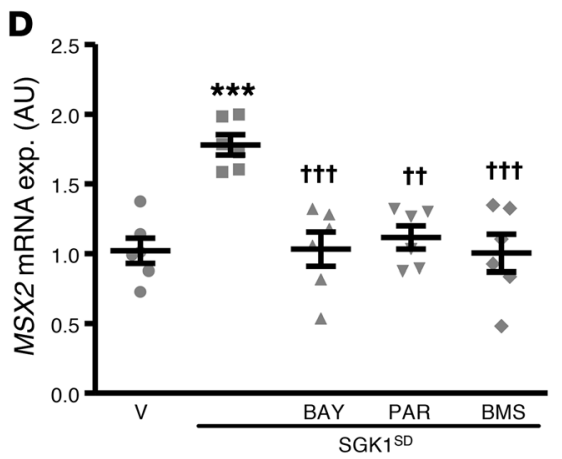
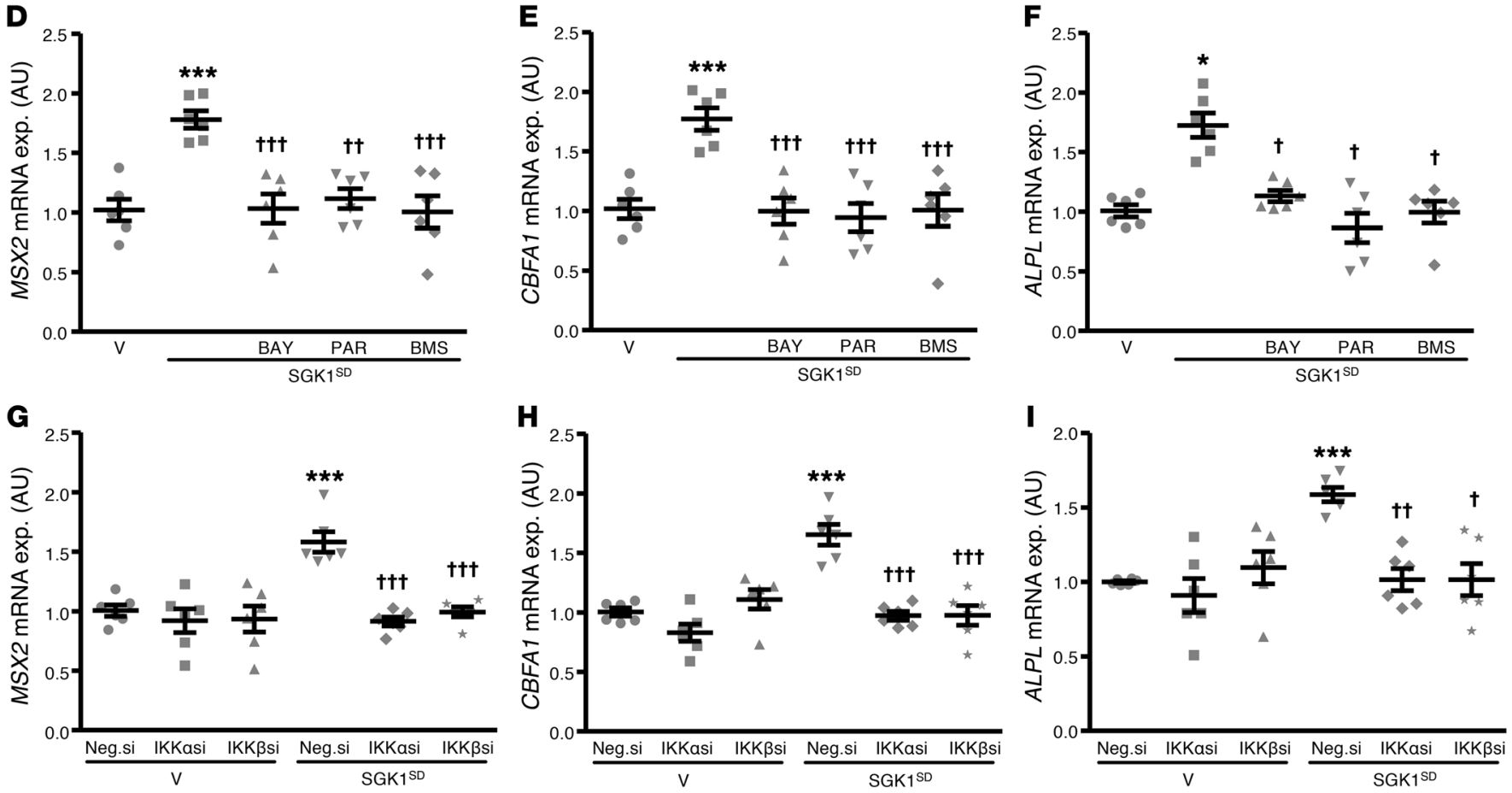

Figure 3. NF-KB-dependent effects of SGK1 on osteo-/chondrogenic signaling in primary HAoSMCs. (A) Representative confocal microscopy images ( $n=3$ per group) showing NF- $\kappa B$ p 65 protein expression and localization in HAoSMCs following transfection with empty vector (V), constitutively active SCK $1^{54220}$ (SGK1 ${ }^{\mathrm{SD}}$ ), or inactive SGK1 ${ }^{\mathrm{K} 127 \mathrm{~N}}\left(\mathrm{SCK} 1^{\mathrm{KN}}\right)$. Green labeling, NF-KB p65 expression; magenta labeling, nuclei. Scale bars: $20 \mu \mathrm{m}$. (B) Scatter dot plots and arithmetic means \pm SEM ( $n=4$ per group; AU) of NF- $\kappa B$-dependent transcriptional activity measured by luciferase reporter assay in HAoSMCs following transfection with NF-KB-responsive luciferase/Renilla constructs and with empty vector (V), constitutively active SCK1 ${ }^{\mathrm{S422D}}$ (SCK $1^{\mathrm{SD}}$ ), or inactive SCK1 ${ }^{\mathrm{K} 27 \mathrm{~N}}\left(\mathrm{SCK} 1^{\mathrm{KN}}\right.$ ). (C) Scatter dot plots and arithmetic means \pm SEM ( $n=8$ per group; AU) of ZFP36 relative mRNA expression in HAoSMCs following transfection with empty vector $(V)$, constitutively active SGK1 $1^{\mathrm{S} 422 \mathrm{D}}\left(\mathrm{SGK} 1^{\mathrm{SD}}\right)$, or inactive SCK1 ${ }^{\mathrm{K} 127 \mathrm{~N}}\left(\mathrm{SGK} 1^{\mathrm{KN}}\right)$. (D-F) Scatter dot plots and arithmetic means \pm SEM $(n=6$ per group; AU) of MSX2 (D), CBFA1 (E), and ALPL (F) relative mRNA expression in HAoSMCs following transfection with empty vector (V) or constitutively active SGK1 ${ }^{54220}$ $\left(\mathrm{SGK} \mathrm{1}^{\mathrm{SD}}\right.$ ) and additional treatment with control, BAY11-7082 (BAY), parthenolide (PAR), or BMS-345541 (BMS). (G-I) Scatter dot plots and arithmetic means \pm SEM ( $n=6$ per group; AU) of MSX2 (C), CBFA1 (H), and ALPL (I) relative mRNA expression in HAoSMCs following transfection with empty vector $(V)$ or constitutively active $\mathrm{SGK} 1^{\mathrm{S} 222 \mathrm{D}}\left(\mathrm{SCK} 1^{\mathrm{SD}}\right.$ ) and additional silencing with negative control siRNA (Neg.si), IKK $\alpha$ siRNA (IKK $\left.\alpha s i\right)$, or IKK $\beta$ siRNA (IKK $\beta$ si). ${ }^{*} P<0.05$, ${ }^{* * *} P<0.001$ statistically significant vs. V-transfected HAoSMCs; ${ }^{\dagger} P<0.05,{ }^{\dagger+} P<0.01,{ }^{t+t} P<0.001$ statistically significant vs. SCK $1{ }^{\text {IDD }}$-transfected HAoSMCs (1-way ANOVA with Tukey-HSD post hoc test for $\mathbf{B}-\mathbf{E}$ and $\mathbf{H}$ or with Games-Howell post hoc test for $\mathbf{G}$ and $\mathbf{I}$ and Steel-Dwass method for $\mathbf{F}$ ).

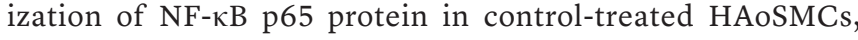
but inhibited the nuclear translocation of $\mathrm{NF}-\kappa \mathrm{B}$ p 65 protein in phosphate-treated HAoSMCs (Figure 5B). Moreover, the phosphate-induced calcium deposition (Figure 5, C and D), alkaline phosphatase activity (Figure 5E), and MSX2, CBFA1, and $A L P L$ mRNA expression (Figure $5, \mathrm{~F}-\mathrm{H}$ ) were in HAoSMCs significantly blunted by additional treatment with the SGK1 inhibitor EMD638683. Treatment with EMD638683 alone did not significantly affect the mineralization and osteo-/chondrogenic signaling in HAoSMCs (Figure 5, C-H).
Accordingly, silencing of the $S G K 1$ gene to inhibit the endogenous expression in HAoSMCs significantly blunted or virtually abrogated the increased calcium content, alkaline phosphatase activity, and osteogenic marker mRNA expression following high-phosphate treatment, without significantly affecting the osteo-/chondrogenic transdifferentiation of control-treated HAoSMCs (Supplemental Figure 4, A-F). These results suggest that SGK1 inhibition dissipates the osteo-/chondrogenic transdifferentiation and calcification of VSMCs in conditions of hyperphosphatemia. 
A

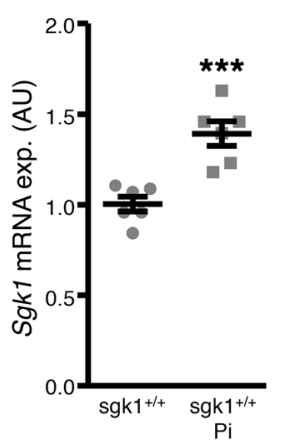

B

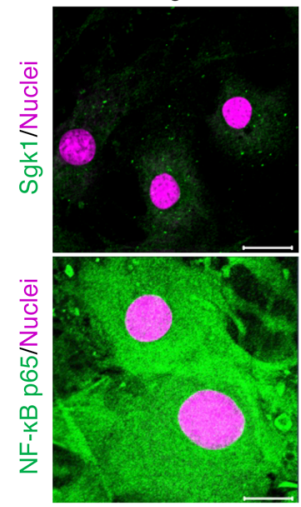

$\mathrm{sgk} 1^{-1-}$

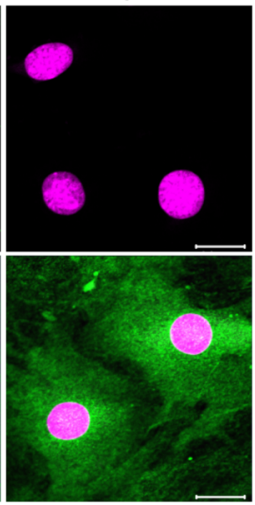

sgk1 ${ }^{+/+} \mathrm{Pi}$

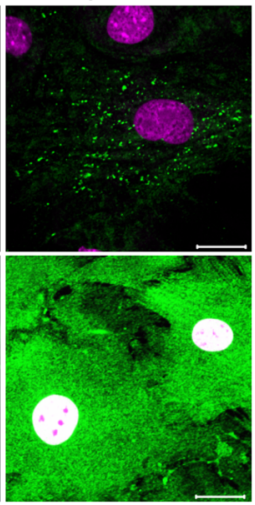

$\operatorname{sgk} 1^{-1-} \mathrm{Pi}$

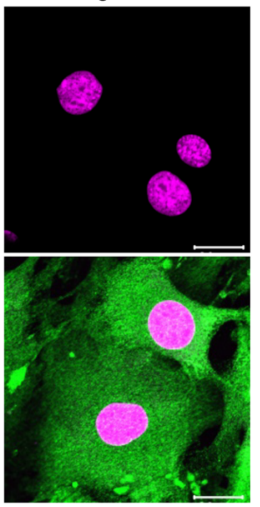

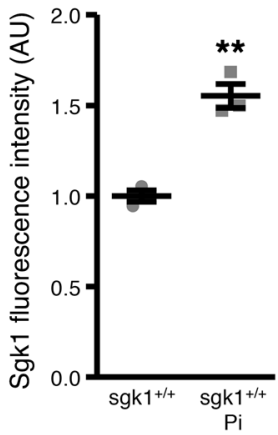

D $\quad \mathrm{sgk}^{+/+}$

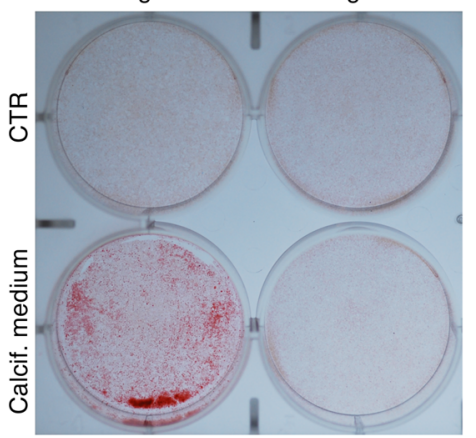

E

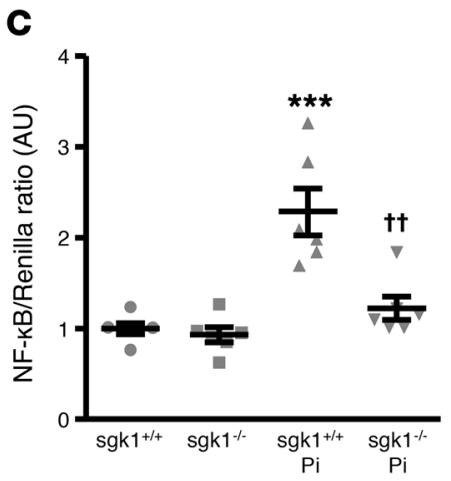

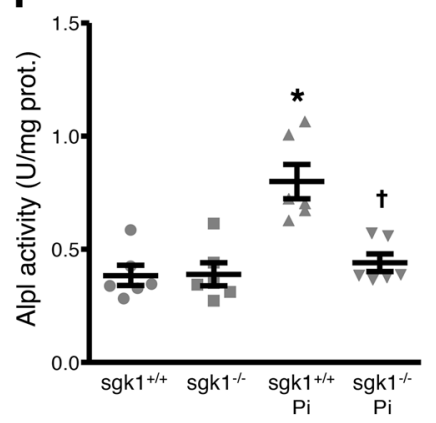

G

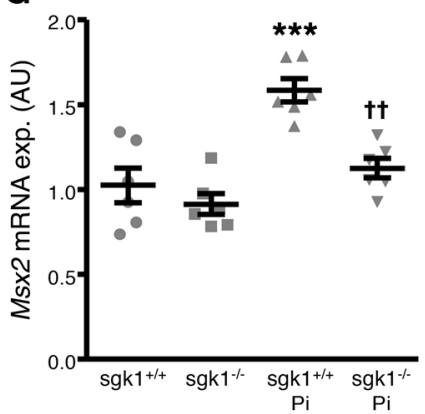

H

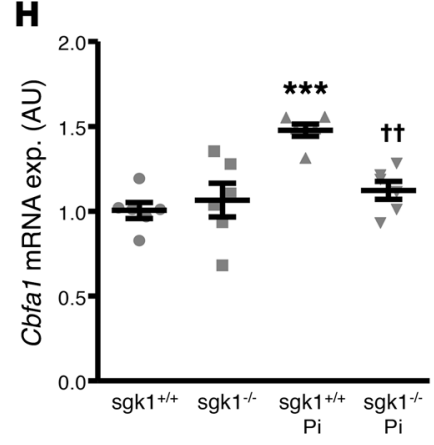

I

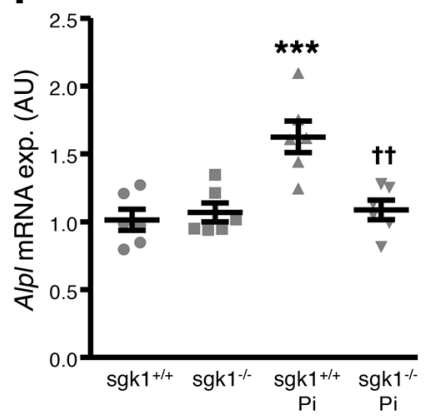

Figure 4. Sgk1 deficiency reduces phosphate-induced osteo-/chondrogenic transdifferentiation and calcification of primary MAoSMCs. (A) Scatter dot plots and arithmetic means \pm SEM ( $n=6$ per group; AU) of Sgk1 relative mRNA expression in primary MAoSMCs isolated from WT mice (sgk $\left.1^{+/+}\right)$and treated with control or $\beta$-glycerophosphate (Pi). (B) Representative confocal microscopy images $(n=3$ per group) showing Sgk1 and NF-kB p65 protein expression and scatter dot plots and arithmetic means \pm SEM ( $n=3$ per group; AU) of normalized Sgk1 fluorescence intensity in sgk1 $1^{-/-}$or sgk $1^{+/+} \mathrm{MAoSMCs}$ treated with control or Pi. Green labeling, Sgk1 or NF-кB p65 expression; magenta labeling, nuclei. Scale bars: $20 \mu \mathrm{m}$. ${ }^{* *} P<0.01,{ }^{* * *} P<0.001$ statistically significant vs. control-treated MAoSMCs (unpaired 2-tailed $t$ test). (C) Scatter dot plots and arithmetic means \pm SEM ( $n=6$ per group; AU) of NF- $\mathrm{kB}$ dependent transcriptional activity measured by luciferase reporter assay in sgk1 $1^{-/-}$or sgk1+/+ MAoSMCs treated with control or Pi. (D) Representative original images ( $n=3$ per group) showing alizarin red staining in sgk $1^{-/-}$or $5 g k 1^{+/+}$MAoSMCs treated with control or calcification medium. Calcified areas are shown as red staining. (E-I) Scatter dot plots and arithmetic means \pm SEM of calcium content $(E, n=6$ per group, $\mu \mathrm{g} / \mathrm{mg}$ protein), alkaline phosphatase activity (F, $n=6$ per group, $\mathrm{U} / \mathrm{mg}$ protein), and $M s \times 2(\mathbf{G}), C b f a 1(\mathbf{H})$, and $A / p l(\mathbf{I})$ relative mRNA expression $\left(n=6\right.$ per group; AU) in sgk1 $1^{-/-}$or sgk $1^{+/+}$ MAoSMCs treated with control or $\mathrm{Pi} .{ }^{*} P<0.05,{ }^{* * *} P<0.001$ statistically significant vs. control-treated sgk $\mathrm{T}^{+/+} \mathrm{MAoSMCs} ;{ }^{\dagger} P<0.05,{ }^{+\dagger} P<0.01,{ }^{\dagger+\dagger} P<0.001$ statistically significant vs. calcification medium/Pi-treated sgk $1^{+/+}$MAoSMCs (1-way ANOVA with Tukey-HSD post hoc test for C, E, and G-I and SteelDwass method for $\mathbf{F}$ ).

In addition, the SGK1 inhibitor EMD638683 partly reduced aldosterone-induced osteogenic marker mRNA expression in HAoSMCs, but it was less effective than the mineralocorticoid receptor antagonist spironolactone (Supplemental Figure 5).

Effect of Sgk1 deficiency during cholecalciferol overload-induced vascular calcification in mice. To investigate the in vivo signifi- cance of Sgk1 in medial vascular calcification, experiments were performed in Sgk1-deficient mice ( $\left.\mathrm{sgk}^{-1^{-}}\right)$and corresponding WT mice $\left(\mathrm{sgk}^{+/+}\right)$following high-dosed cholecalciferol treatment. As a result, cholecalciferol treatment significantly increased plasma calcium, FGF23 C-term, aldosterone, and cortisol levels to similarly high values in both sgk1 $1^{-/}$mice and $\mathrm{sgk} 1^{+/+}$mice (Supplemen- 
A

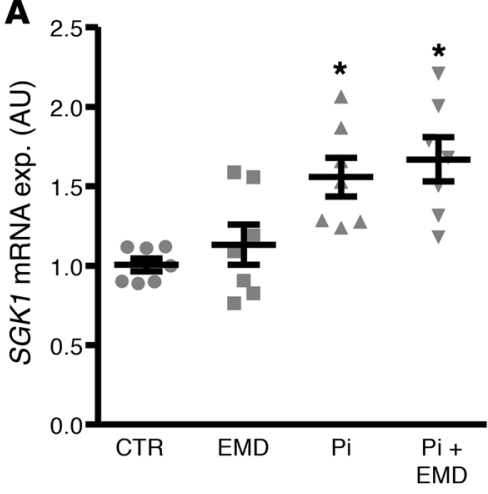

C

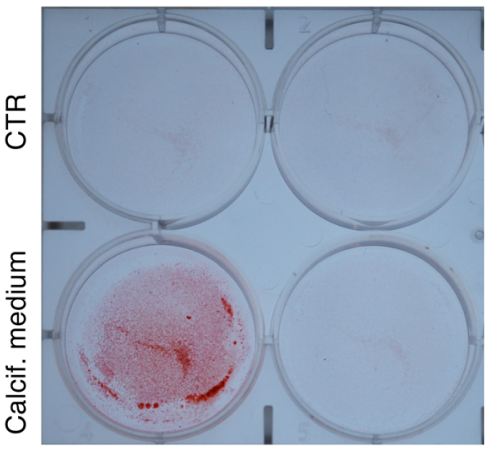

F

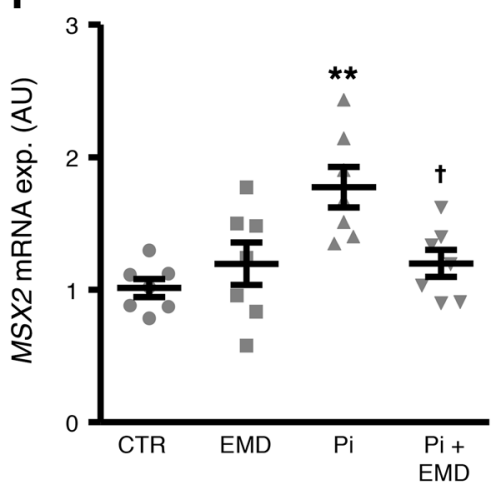

B

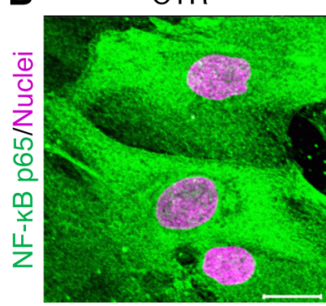

EMD

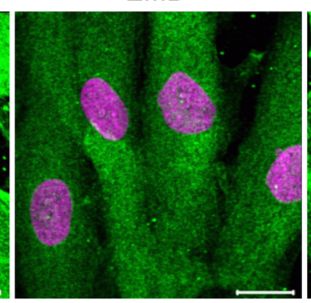

$\mathrm{Pi}$

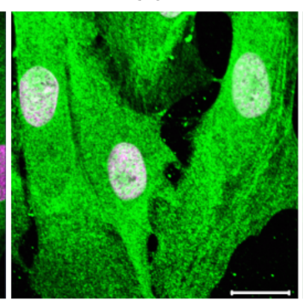

$P i+E M D$

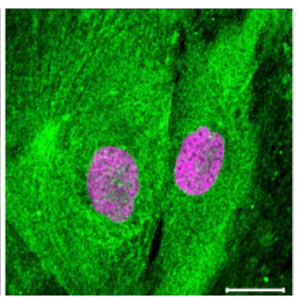

E

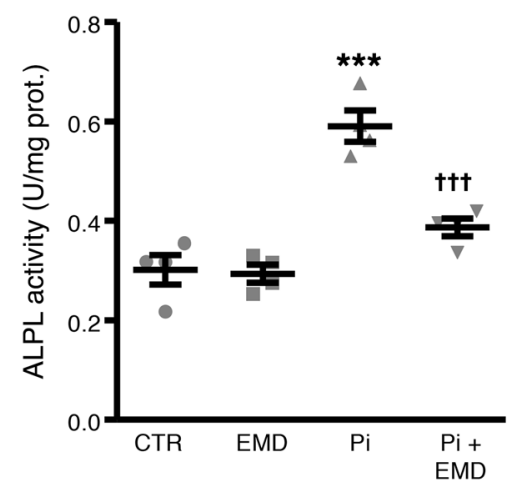

H
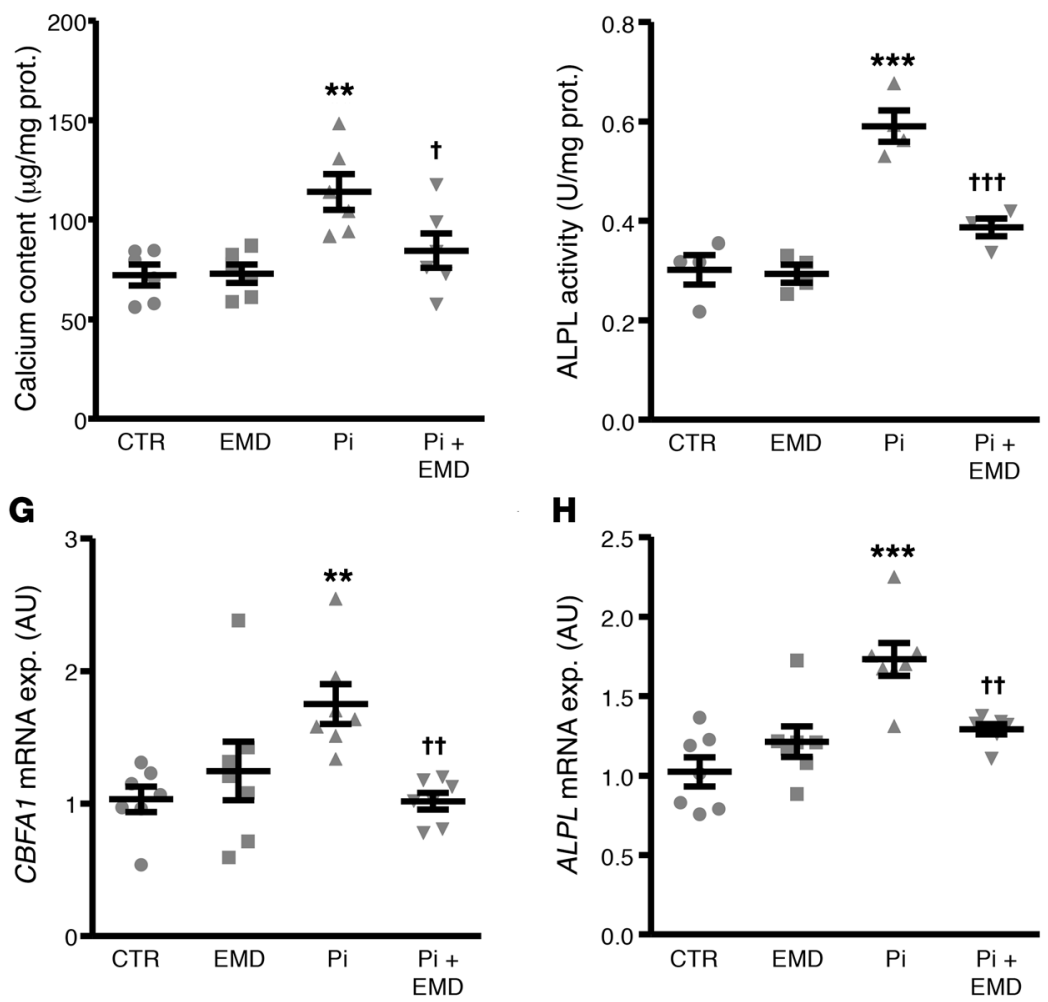

Figure 5. SGK1 inhibition ameliorates phosphate-induced osteo-/chondrogenic transdifferentiation and calcification of primary HAoSMCs. (A) Scatter dot plots and arithmetic means \pm SEM ( $n=7$ per group; AU) of SGK1 relative mRNA expression in HAoSMCs following treatment with control or $\beta$-glycerophosphate $(\mathrm{Pi})$ without or with additional treatment with SGK1 inhibitor EMD638683 (EMD). (B) Representative confocal microscopy images ( $n=3$ per group) showing NF-אB p65 protein expression and localization in HAoSMCs following treatment with control or $\beta$-glycerophosphate (Pi) without or with additional treatment with SGK1 inhibitor EMD638683 (EMD). Green labeling, NF-кB p65 expression; magenta labeling, nuclei. Scale bars: $20 \mu \mathrm{m}$. (C) Representative original images ( $n=4$ per group) showing alizarin red staining in HAoSMCs following treatment with control or with calcification medium without or with additional treatment with SGK1 inhibitor EMD638683 (EMD). Calcified areas are shown as red staining. (D-H) Scatter dot plots and arithmetic means \pm SEM of calcium content ( $D, n=6$ per group, $\mu \mathrm{g} / \mathrm{mg}$ protein), alkaline phosphatase activity $(\mathbf{E}, n=4$ per group, $U / \mathrm{mg}$ protein), and $M S X 2$ (F), CBFA1 (G), and ALPL (H) relative mRNA expression ( $n=7$ per group; AU) in HAoSMCs following treatment with control or phosphate (Pi) without or with additional treatment with SGK1 inhibitor EMD638683 (EMD). ${ }^{*} P<0.05,{ }^{* *} P<0.01,{ }^{* *} P<0.001$ statistically significant vs. control-treated HAoSMCs; ${ }^{\dagger} P<0.05,{ }^{t \dagger} P<0.01,{ }^{t+t} P<0.001$ statistically significant vs. HAoSMCs treated with calcification medium/Pi alone (Steel-Dwass method for $\mathbf{A}$ and 1 -way ANOVA with Tukey-HSD post hoc test for $\mathbf{D}-\mathbf{H}$ ).

tal Table 2). Plasma phosphorus concentrations were not significantly modified following high-dosed cholecalciferol treatment (Supplemental Table 2).

As shown by alizarin red staining and quantification of aortic arch calcium content, high-dosed cholecalciferol treatment induced aortic calcification in $\mathrm{sgk}^{+/+}$mice. Cholecalciferol- induced vascular calcification was significantly blunted in aortae of $\mathrm{sgk1}^{-/-}$mice (Figure 6, A and B). The aortic pulse propagation velocity, as a marker of vascular stiffness, was increased after cholecalciferol treatment in $\mathrm{sgk}^{+/+}$mice, an effect blunted in $\mathrm{sgk} 1^{-/-}$mice (Figure 6C). Similarly, the mRNA expression of stiffness markers collagen type I (Col1a1) and fibronectin (Fn1) 
A

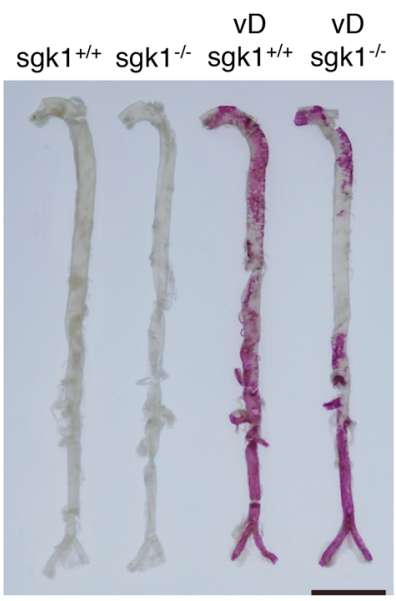

D

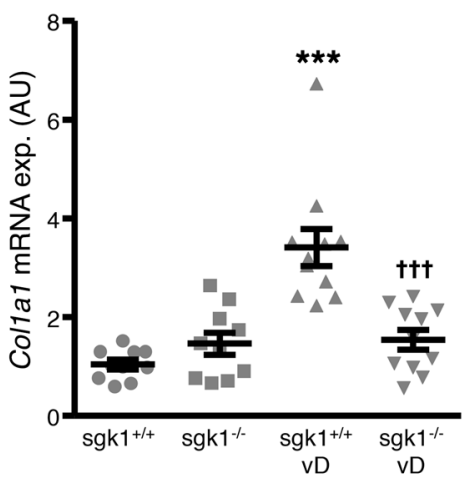

B

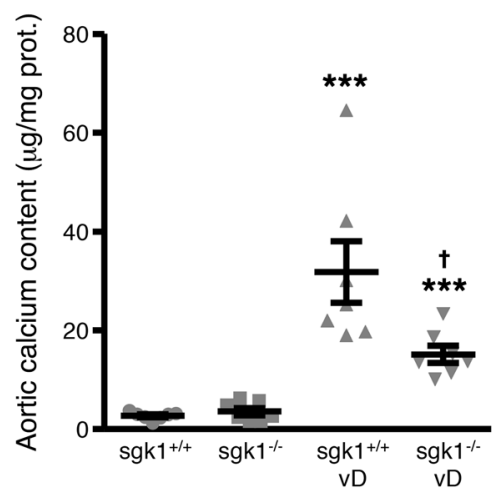

E

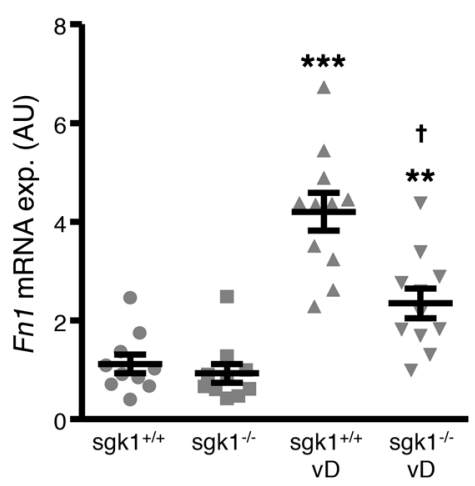

C

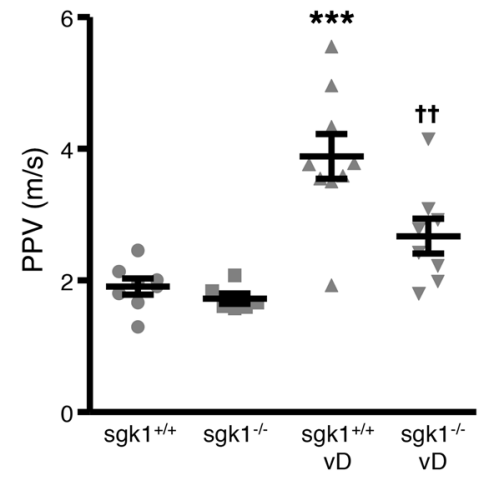

$\mathbf{F}$

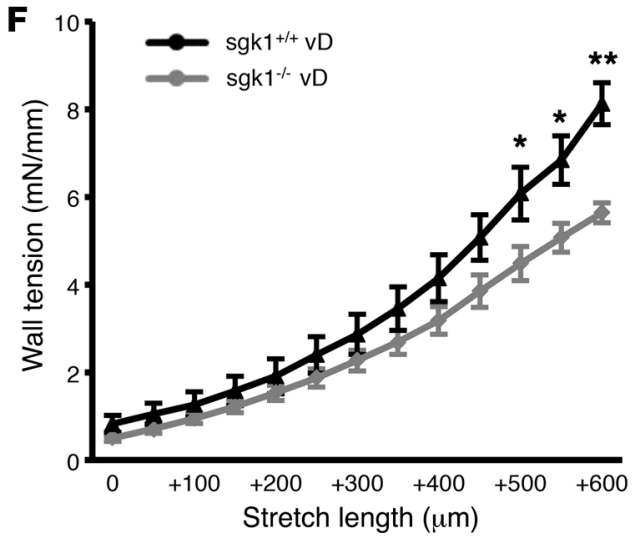

Figure 6. Sgk1 deficiency ameliorates vascular calcification and stiffness during vitamin $\mathbf{D}_{3}$ overload-induced calcification. (A) Representative original images ( $n=$ at least 3 per group) showing aortic alizarin red staining in Sgk1-deficient mice (sgk $1^{-/-}$) or corresponding WT mice (sgk $1^{+/+}$) receiving vehicle or high-dosed cholecalciferol (VD). Scale bar: $5 \mathrm{~mm}$. Calcified areas are shown as red staining. (B) Scatter dot plots and arithmetic means \pm SEM ( $n=7$ per group; $\mu \mathrm{g} / \mathrm{mg}$ protein) of calcium content in the aortic arch of $\mathrm{sgk} 1^{-1-}$ mice or $\mathrm{sgk} 1^{+/+}$mice receiving vehicle or high-dosed cholecalciferol (vD). (C) Scatter dot plots and arithmetic means \pm SEM ( $n=6-9$ per group; $\mathrm{m} / \mathrm{s}$ ) of abdominal aortic pulse propagation velocity (PPV) in sgk $1^{-/-}$mice or sgk $1^{+/+}$mice receiving vehicle or high-dosed cholecalciferol (vD). (D and E) Scatter dot plots and arithmetic means \pm SEM ( $n=10-11$ per group; AU) of Col1a1 (D) and Fn1 (E) relative mRNA expression in aortic tissue of sgk1 $1^{-/-}$mice or $\mathrm{sgk} 1^{+/+}$mice receiving vehicle or high-dosed cholecalciferol (vD). ${ }^{* *} P<0.01,{ }^{* * *} P<0.001 \mathrm{statistically}$ significant vs. control sgk $1^{+/+}$mice; ${ }^{\dagger} P<0.05,{ }^{\dagger+} P<0.01,{ }^{+\dagger+} P<0.001$ statistically significant vs. vD-treated sgk $1^{+/+}$mice (1-way ANOVA with Tukey-HSD post hoc test for $\mathbf{B}, \mathbf{C}$, and $\mathbf{E}$ or with Games-Howell post hoc test for $\mathbf{D}$ ). (F) Summary data of wall tension ( $n=7$ rings, $4 \mathrm{mice}$ per group; $\mathrm{mN} / \mathrm{mm}$ ) during mechanical stretch $(\mu \mathrm{m})$ ex vivo of abdominal aortae isolated from sgk $1^{-1-}$ mice or sgk1 ${ }^{+/+}$mice receiving high-dosed cholecalciferol $(\mathrm{vD}) .{ }^{*} P<0.05,{ }^{* *} P<$ 0.01 statistically significant vs. vD-treated sgk1 $1^{-/-}$mice (unpaired 2-tailed $t$ test).

was significantly higher in aortic tissue of cholecalciferol-treated $\mathrm{sgk} 1^{+/+}$mice, effects again significantly reduced in aortic tissue of $\mathrm{sgk1}^{-/-}$mice (Figure 6, D and E). Accordingly, after cholecalciferol treatment, aortic rings from $\mathrm{sgk1} 1^{-/}$mice developed less wall tension after ex vivo mechanical stretch as compared with aortic rings from $\mathrm{sgk} 1^{+/+}$mice, indicative of higher aortic stiffness in $\mathrm{sgk} 1^{+/+}$ mice (Figure 6F). No differences between sgk $1^{+/+}$and sgk $1^{-/}$control treated mice were observed (Supplemental Figure 6).

In sgk $1^{+/+}$mice, cholecalciferol treatment significantly upregulated aortic Sgk1 mRNA and protein expression (Figure 7, A and B). As expected, no Sgk1 expression was observed in the aortic media of $\operatorname{sgk1} 1^{--}$mice (Figure 7A). The protein abundance of Msx2 in the media of aortic sections (Figure 7A), and Msx2, Wnt7a, Wnt3a, Cbfa1, and Alpl mRNA expression in the aortic tissue (Figure 7, C-G), were all significantly higher following cholecalciferol overload in sgk $1^{+/+}$mice as compared with control-treated mice, effects again significantly blunted in the sgk $1^{-/}$mice. The effects on aortic osteogenic signaling and calcification were paralleled by upregulation of $Z f p 36$ mRNA expression (Figure $7 \mathrm{H}$ ) and downregulation of Ankh mRNA levels (Figure 7I) in cholecalciferol-treated sgk $1^{1 /+}$ mice, but not sgk1 $1^{-/}$mice. Thus, Sgk1 deficiency reduces aortic osteoinduction, vascular calcification, and vascular stiffening in the cholecalciferol overload mouse model.

Effect of Sgk1 inhibition during cholecalciferol overload-induced vascular calcification in mice. To explore whether Sgk1 inhibition shows similar protective effects on vascular calcification in vivo, C57BL/6 mice were treated with high-dosed cholecalciferol without or with additional treatment with the Sgk1 inhibitor EMD638683. The increased plasma calcium, FGF23 C-term, and cortisol levels in cholecalciferol-treated mice were not significantly modified following additional treatment with EMD638683 (Supplemental Table 3). Plasma aldosterone levels tended to be higher in cholecalciferol-treated mice than in control mice and not significantly affected by EMD638683 (Supplemental Table 3). Plasma phos- 
A
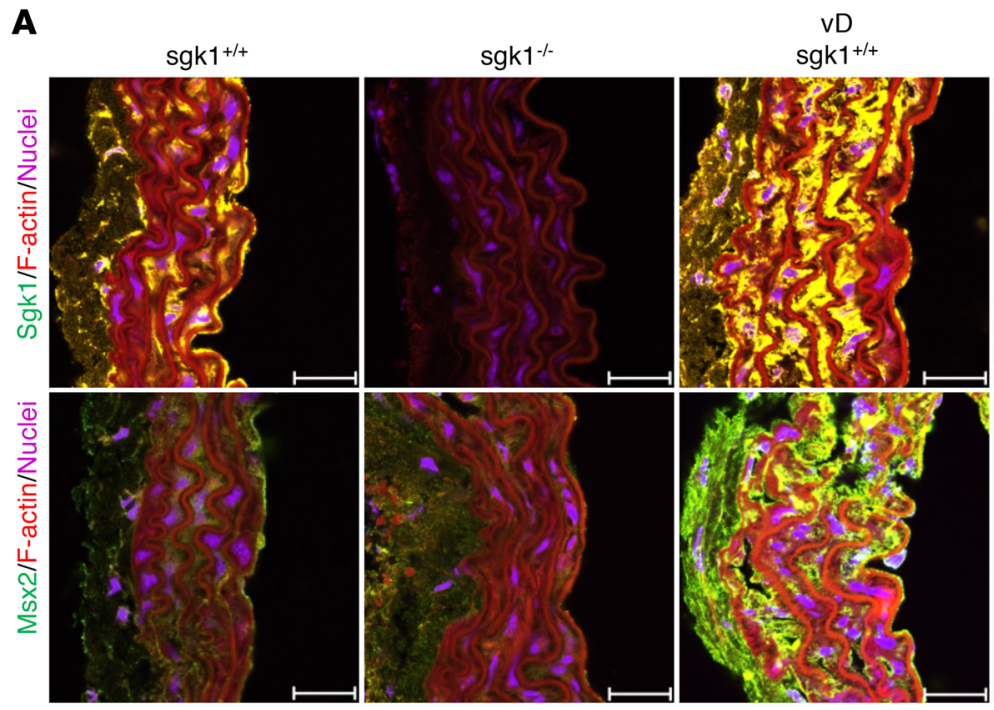
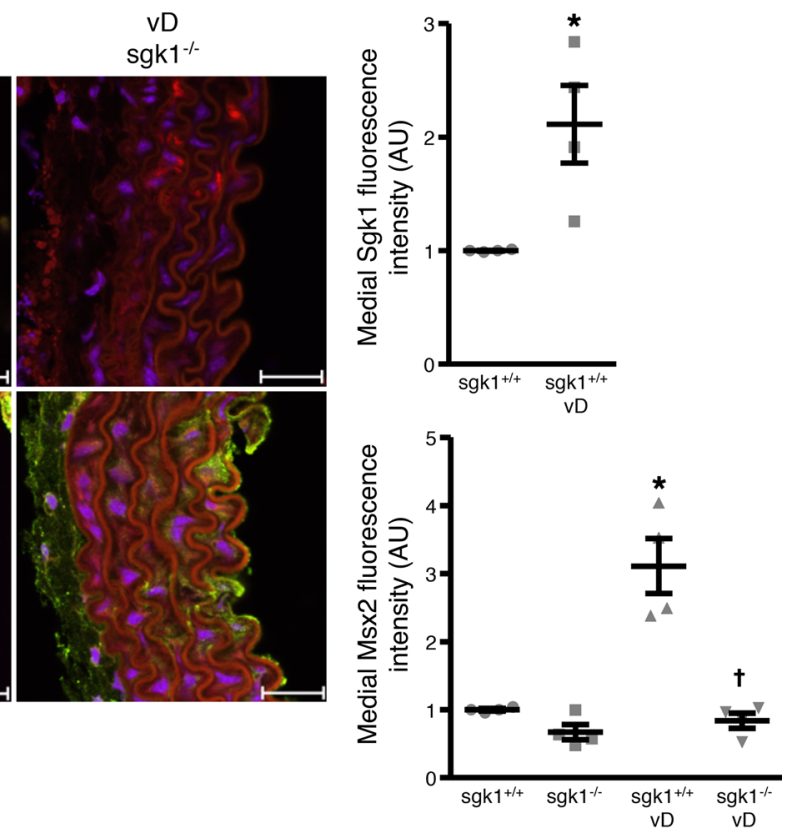

B
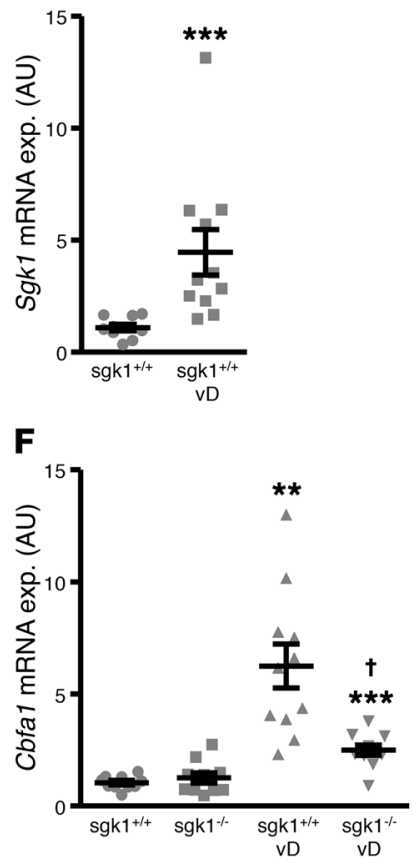

C

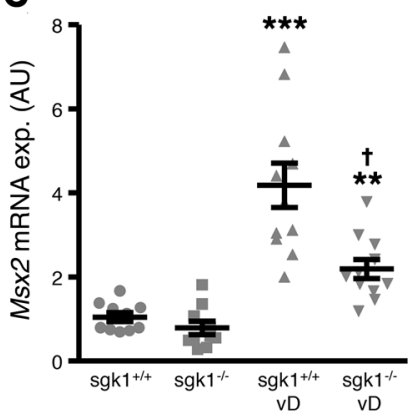

G

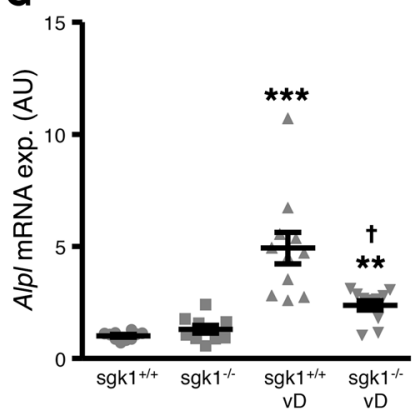

D

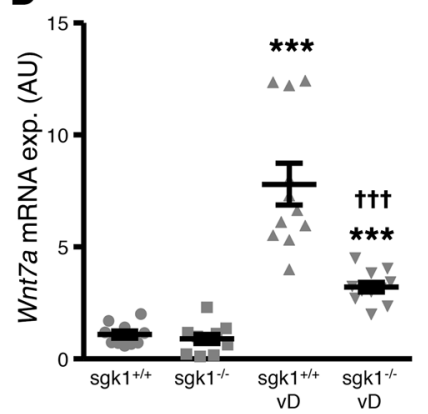

H

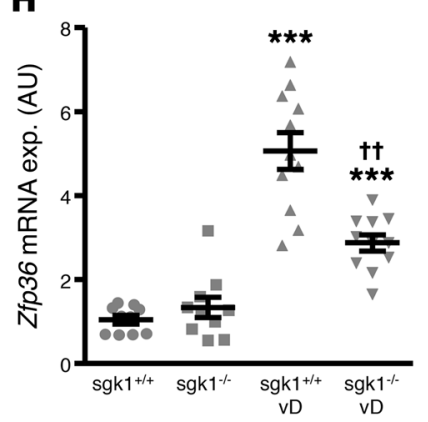

E

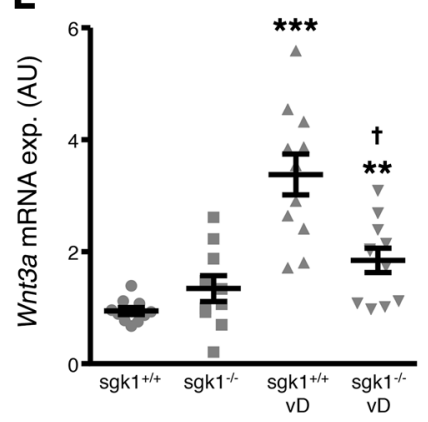

I

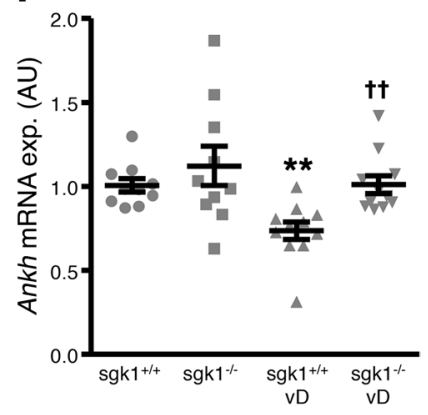

Figure 7. Sgk1 deficiency ameliorates vascular osteoinductive signaling during vitamin $\mathbf{D}_{3}$ overload-induced calcification. (A) Representative confocal microscopy images ( $n=4$ per group) showing Sgk1 and Msx2 protein expression and scatter dot plots and arithmetic means \pm SEM ( $n=4$ per group; AU) of normalized Sgk1 and Msx2 fluorescence intensity in aortic tissues of Sgk1-deficient mice (sgk $1^{-/-}$) or corresponding WT mice (sgk $1^{+/+}$) receiving vehicle or high-dosed cholecalciferol (vD). Green labeling, protein expression; magenta labeling, nuclei; red labeling, actin staining. Scale bars: $25 \mu \mathrm{m}$. (B) Scatter dot plots and arithmetic means \pm SEM ( $n=10-11$ per group; AU) of Sgk1 relative mRNA expression in aortic tissue of sgk $1^{+/+}$mice receiving vehicle or highdosed cholecalciferol (vD). (C-I) Scatter dot plots and arithmetic means \pm SEM ( $n=10-11$ per group; AU) of Msx2 (C), Wnt7a (D), Wnt3a (E), Cbfa1 (F), Alpl (C), Zfp36 (H), and Ankh (I) relative mRNA expression in aortic tissue of sgk1 $1^{-/-}$mice or sgk1+/+ mice receiving vehicle or high-dosed cholecalciferol (vD). ${ }^{*} P<0.05,{ }^{* *} P<0.01,{ }^{* *} P<0.001$ statistically significant vs. control sgk ${ }^{+/+}$mice; ${ }^{\dagger} P<0.05,{ }^{{ }^{+}} P<0.01,{ }^{t+\dagger} P<0.001$ statistically significant vs. vD-treated $\mathrm{sgk}^{+/+}$mice (unpaired 2-tailed $t$ test for Sgk1 panel of $\mathbf{A}$ and for B; 1-way ANOVA with Games-Howell post hoc test for Msx2 panel of $\mathbf{A}$ and for $\mathbf{C}-\mathbf{F}$; and Steel-Dwass method for G-I).

phorus levels were not significantly different between the groups (Supplemental Table 3).

Cholecalciferol overload induced aortic calcification in mice, an effect significantly ameliorated following additional treatment with EMD638683 (Figure 8, A and B). Furthermore, the increased aortic pulse propagation velocity after cholecalciferol treatment in mice was significantly reduced by EMD638683 (Figure 8C), suggesting lower aortic stiffness following Sgk1 inhibition. These 
A
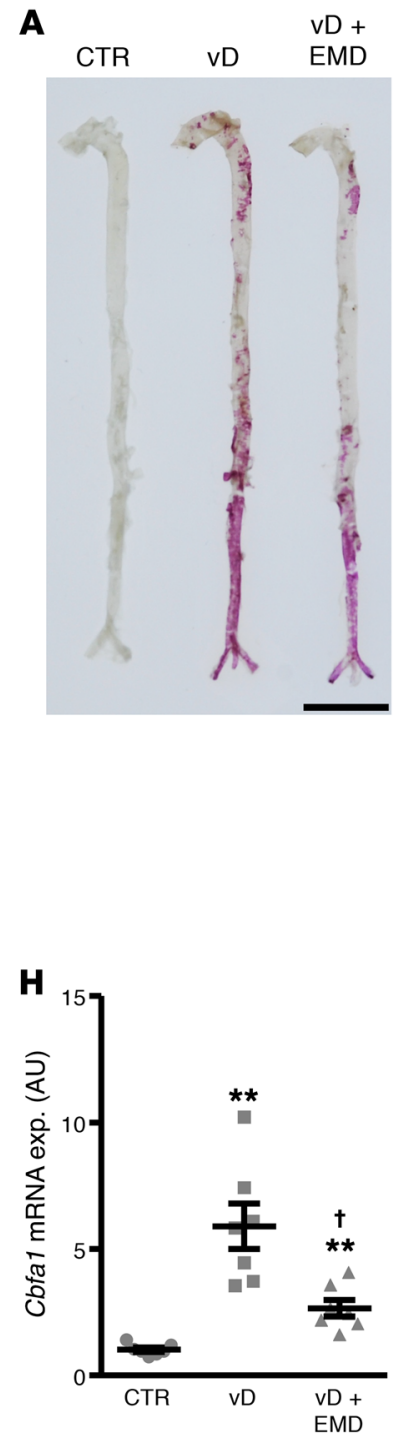

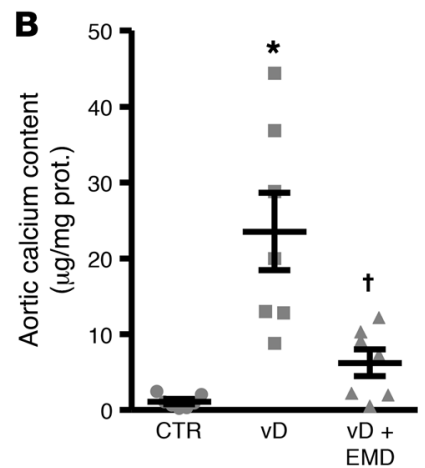

E

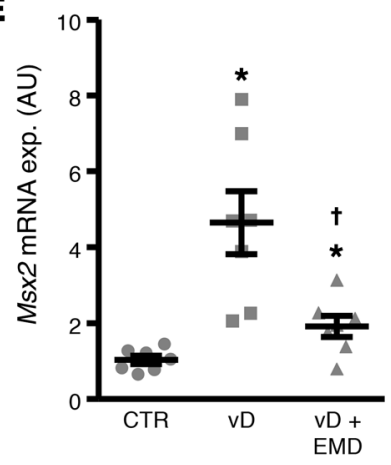

I

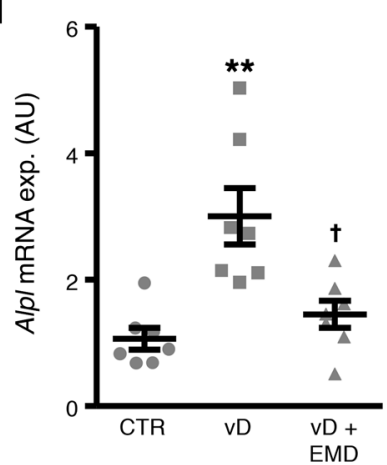

C

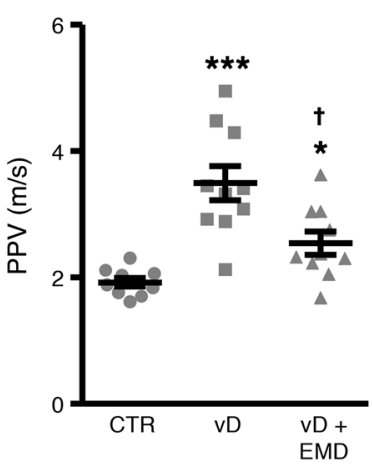

$\mathbf{F}$

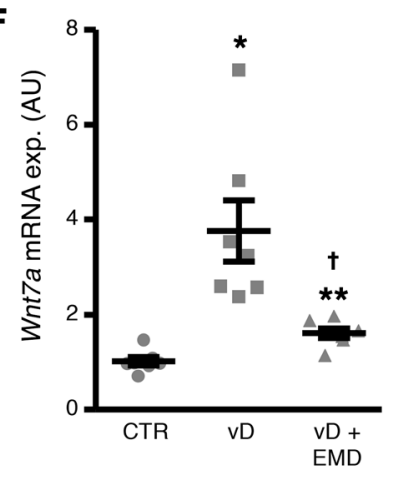

J

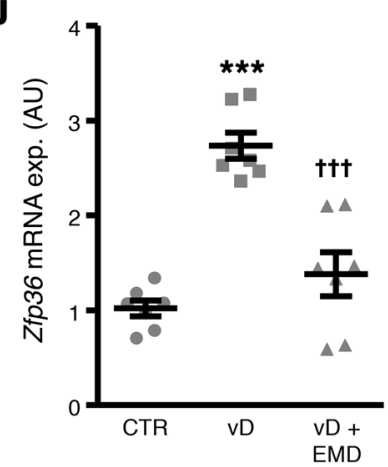

D

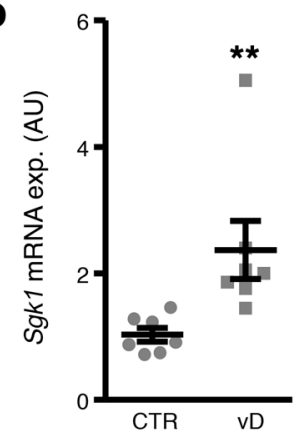

G

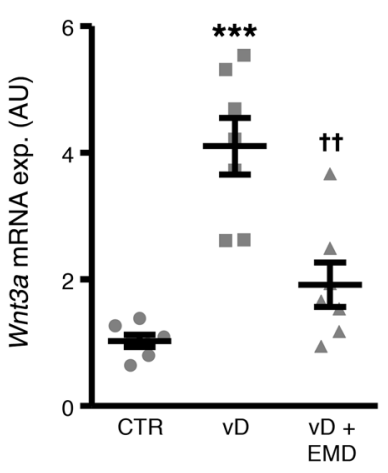

$\mathbf{K}$

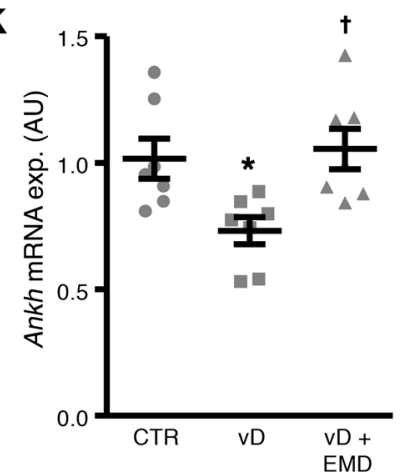

Figure 8. Sgk1 inhibition ameliorates vascular calcification, stiffness, and osteoinductive signaling during vitamin $D_{3}$ overload-induced calcification. (A) Representative original images ( $n=3$ per group) showing aortic alizarin red staining in mice receiving vehicle or high-dosed cholecalciferol ( $\mathrm{VD}$ ) without or with additional treatment with SGK1 inhibitor EMD638683 (EMD). Scale bar: $5 \mathrm{~mm}$. The calcified areas are shown as red staining. (B) Scatter dot plots and arithmetic means \pm SEM ( $n=7$ per group; $\mu \mathrm{g} / \mathrm{mg}$ protein) of calcium content in the aortic arch of mice receiving vehicle or high-dosed cholecalciferol (VD) without or with additional treatment with SGK1 inhibitor EMD638683 (EMD). (C) Scatter dot plots and arithmetic means \pm SEM ( $n=10$ per group; $\mathrm{m} / \mathrm{s}$ ) of abdominal aortic pulse propagation velocity (PPV) in mice receiving vehicle or high-dosed cholecalciferol (vD) without or with additional treatment with SGK1 inhibitor EMD638683 (EMD). (D) Scatter dot plots and arithmetic means \pm SEM ( $n=7$ per group; AU) of Sgk1 relative mRNA expression in aortic tissue of mice receiving vehicle or high-dosed cholecalciferol (vD). (E-K) Scatter dot plots and arithmetic means \pm SEM ( $n=7$ per group; AU) of Msx2 (E), Wnt7a (F), Wnt3a (C), Cbfa1 (H), Alpl (I), Zfp36 (J), and Ankh (K) relative mRNA expression in aortic tissue of mice receiving vehicle or high-dosed cholecalciferol (VD) without or with additional treatment with SGK1 inhibitor EMD638683 (EMD). ${ }^{*} P<0.05,{ }^{* *} P<0.01,{ }^{* * *} P<0.001$ statistically significant vs. control mice; ${ }^{\dagger} P<0.05,{ }^{+\dagger} P<0.01,{ }^{+\dagger+} P<0.001$ statistically significant vs. vD-treated mice (unpaired 2-tailed $t$ test for $\mathbf{D}$ and 1-way ANOVA with Tukey-HSD post hoc test for $\mathbf{J}$ and $\mathbf{K}$ or with Games-Howell post hoc test for $\mathbf{B}, \mathbf{C}$, and $\mathbf{E}-\mathbf{I}$ ).

effects were paralleled by similar inhibition of aortic osteogenic signaling. Cholecalciferol treatment significantly upregulated Sgk1 mRNA expression and caused increased mRNA expression of the osteogenic markers and Zfp36 mRNA as well as downregulated Ankh mRNA levels in aortic tissue as compared with control-treated mice (Figure 8, D-K). These effects were blunted by EMD638683 treatment (Figure 8, E-K). Taken together, these observations indicate that Sgk1 inhibition by EMD638683 ameliorates aortic osteoinductive signaling, vascular calcification, and vascular stiffness in the cholecalciferol overload mouse model.
Effect of Sgk1 deficiency on vascular calcification in murine renal failure. Additional experiments investigated the effect of Sgk1 deficiency in a clinically more relevant model of renal failure. To this end, subtotal nephrectomy was performed in Sgk1-deficient mice $\left(\mathrm{sgk} 1^{--}\right)$and corresponding WT mice $\left(\mathrm{sgk}^{+/+}\right)$on an apolipoprotein E-deficient background (apoE ${ }^{-1-}$ ). Subtotal nephrectomy increased blood urea nitrogen (BUN) to similar values in both genotypes, confirming comparability of the groups (Supplemental Table 4). After 6 weeks of subtotal nephrectomy, BUN levels tended to increase in apoE $\mathrm{E}^{-/-} \mathrm{sgk1}^{+/+}$mice to higher levels than in 
A

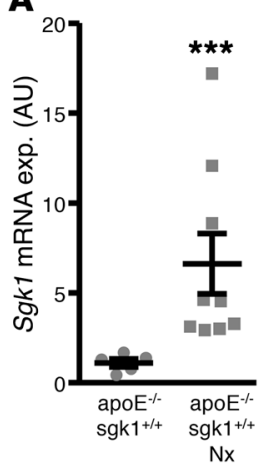

B

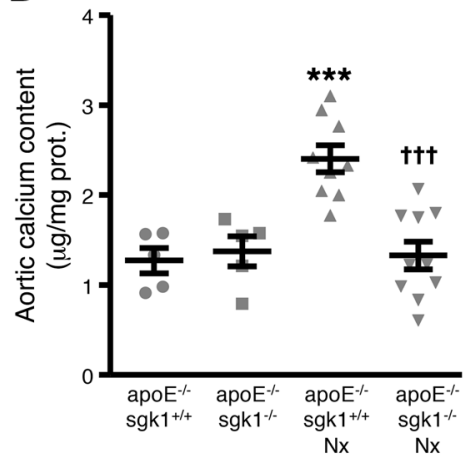

C

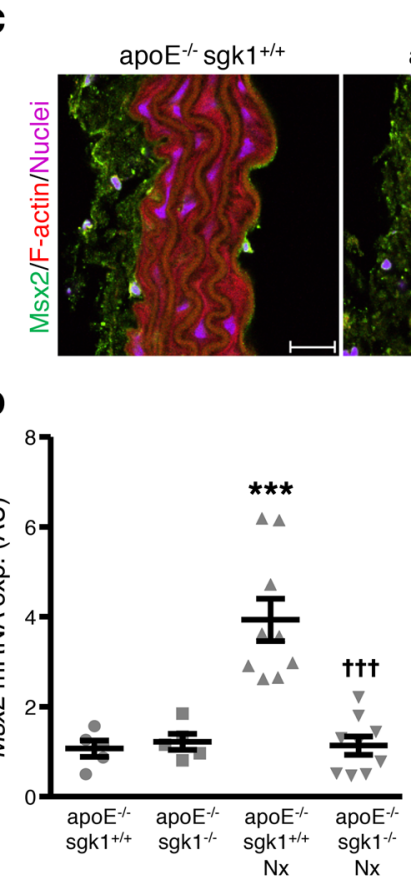

apoE ${ }^{-1}$ sgk $1^{-1}$

$\mathrm{Nx}$ apoE ${ }^{-/-} \mathrm{sgk}^{+/+}$
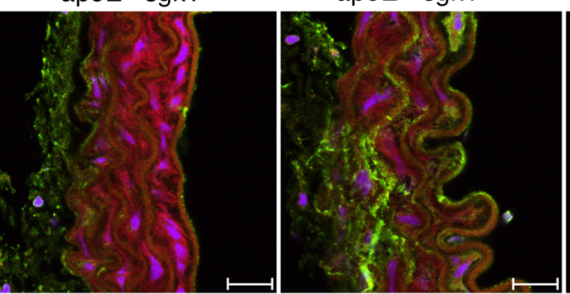

$\mathrm{Nx}$ $\mathrm{apoE}^{-/-} \mathrm{sgk} 1^{-/-}$
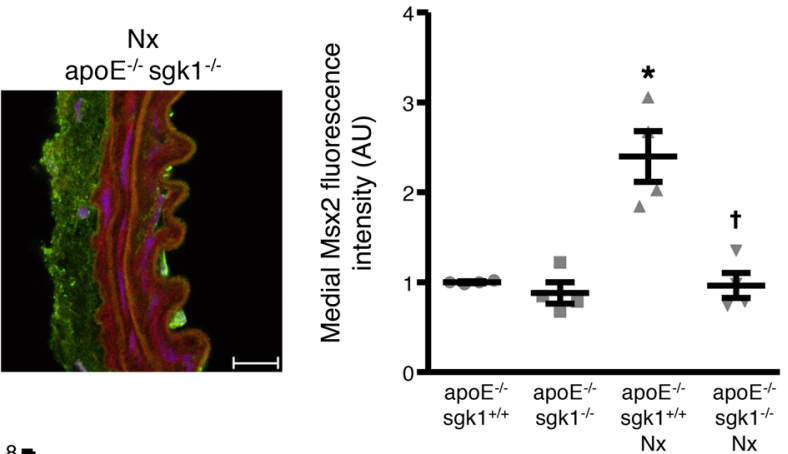

E
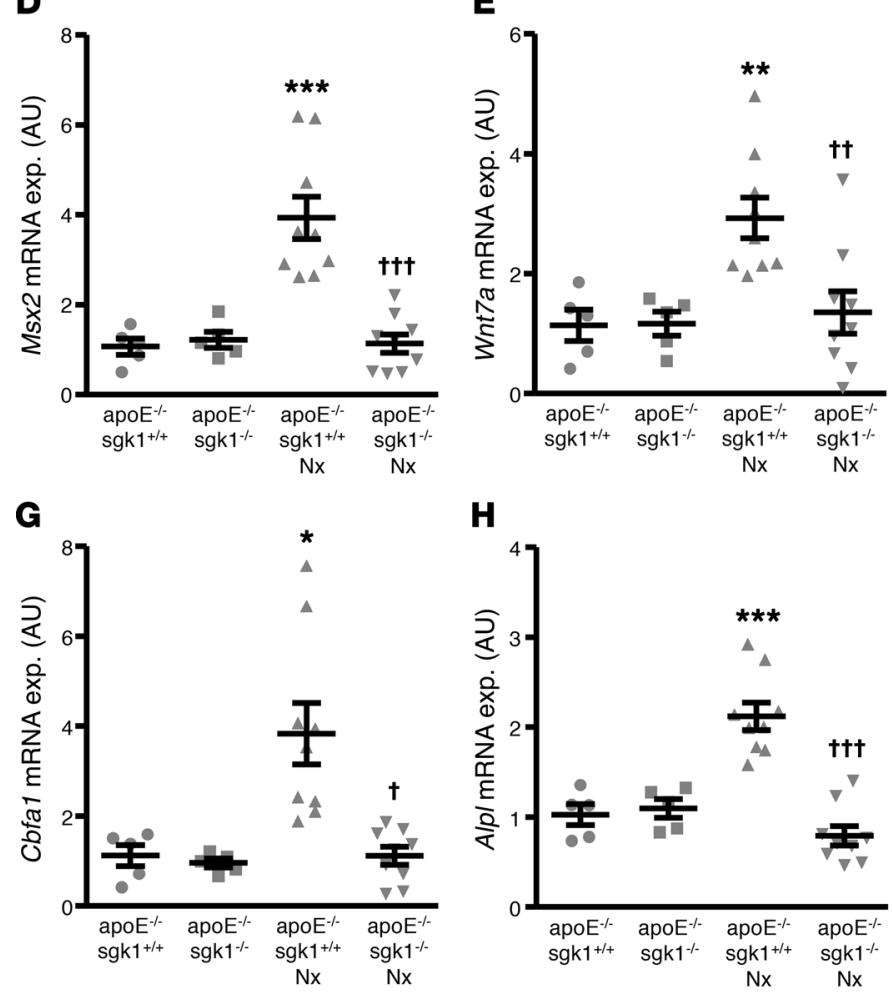

F

H
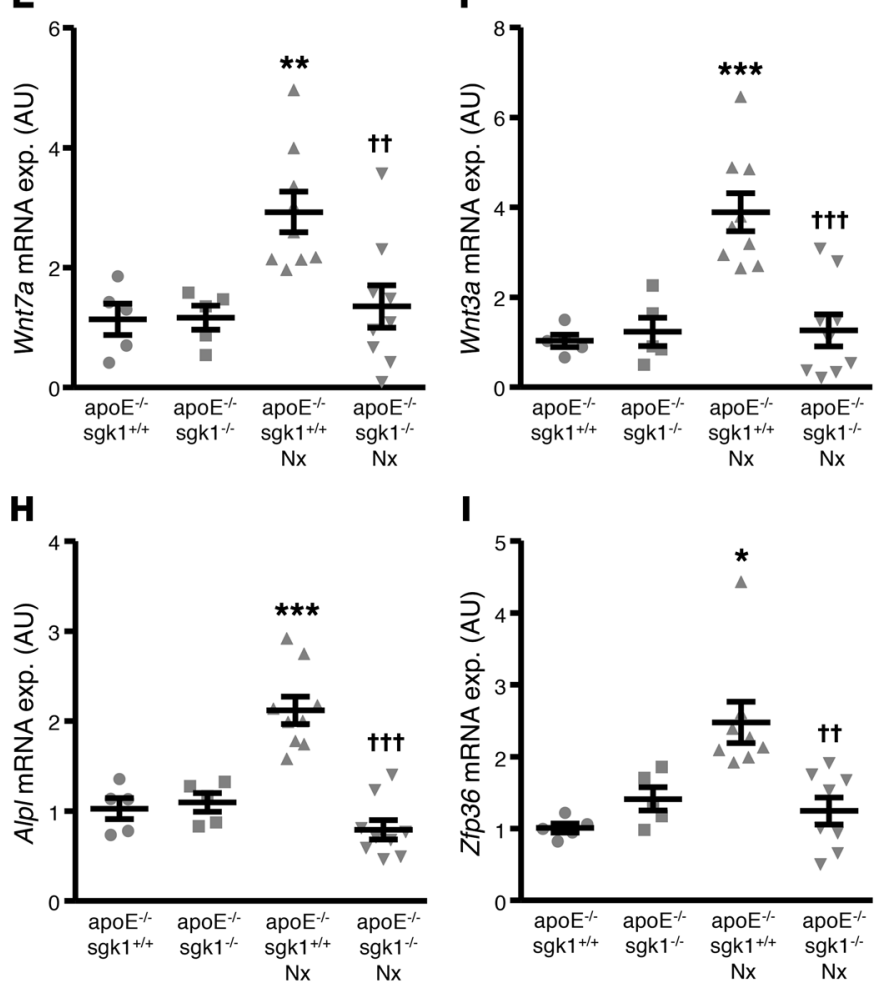

I

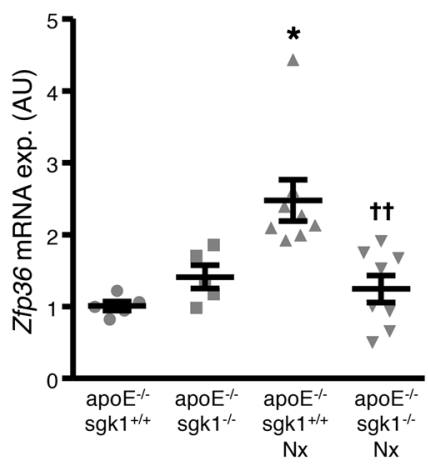

$\mathbf{J}$

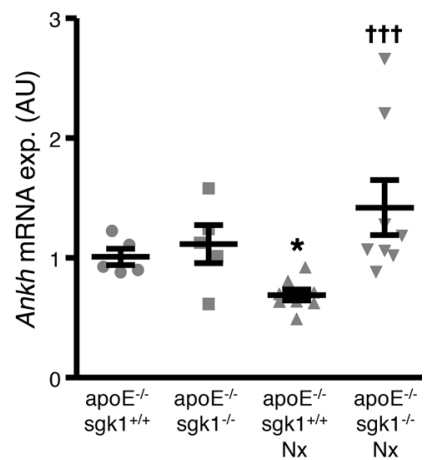

Figure 9. Sgk1 deficiency ameliorates vascular calcification in apolipoprotein E-null mice after subtotal nephrectomy. (A) Scatter dot plots and arithmetic means \pm SEM ( $n=5-9$ per group; AU) of $S g k 1$ relative mRNA expression in aortic tissue of mice lacking apolipoprotein $E$ (apoE $E^{-1}$ ) without or with subtotal nephrectomy $(\mathrm{Nx}){ }^{* * *} P<0.001$ statistically significant vs. control mice (unpaired 2 -tailed $t$ test). (B) Scatter dot plots and arithmetic means $\pm \mathrm{SEM}$

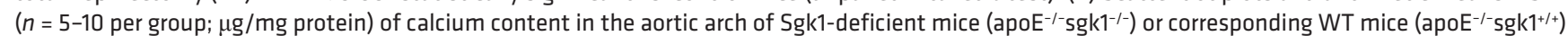
under the apoE $\mathrm{E}^{-1-}$ background without or with subtotal nephrectomy $(\mathrm{Nx})$. (C) Representative confocal microscopy images ( $n=4$ per group) showing Msx2 protein expression and scatter dot plots and arithmetic means \pm SEM ( $n=4$ per group; AU) of normalized Msx2 fluorescence intensity in aortic tissues of apoE $E^{-1-} \mathrm{sgk}^{-1-}$ mice or corresponding apoE $\mathrm{E}^{-1-} \mathrm{ggk}^{+/+}$mice without or with subtotal nephrectomy ( $\mathrm{Nx}$ ). Green labeling, Msx2 expression; magenta labeling, nuclei; red labeling, actin staining. Scale bars: $25 \mu \mathrm{m}$. (D-J) Scatter dot plots and arithmetic means \pm SEM ( $n=5-9$ per group; AU) of Msx2 (D), Wnt7a (E),

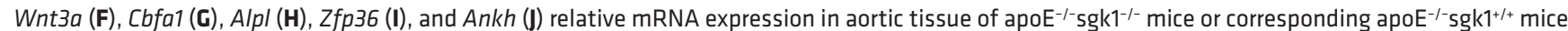
without or with subtotal nephrectomy $(\mathrm{Nx}) .{ }^{*} P<0.05,{ }^{* *} P<0.01$, ${ }^{* * *} P<0.001$ statistically significant vs. control apoE ${ }^{-/-}$sgk ${ }^{+/+}$mice; ${ }^{\dagger} P<0.05,{ }^{+t} P<0.01$, ${ }^{t+t} P<0.001$ statistically significant vs. Nx-treated apoE ${ }^{-/-}$sgk1 ${ }^{+/+}$mice (1-way ANOVA with Tukey-HSD post hoc test for B, D-F, H, and J or with Games-Howell post hoc test for $\mathbf{C}$ and $\mathbf{G}$, and Steel-Dwass method for $\mathbf{I}$ ). 

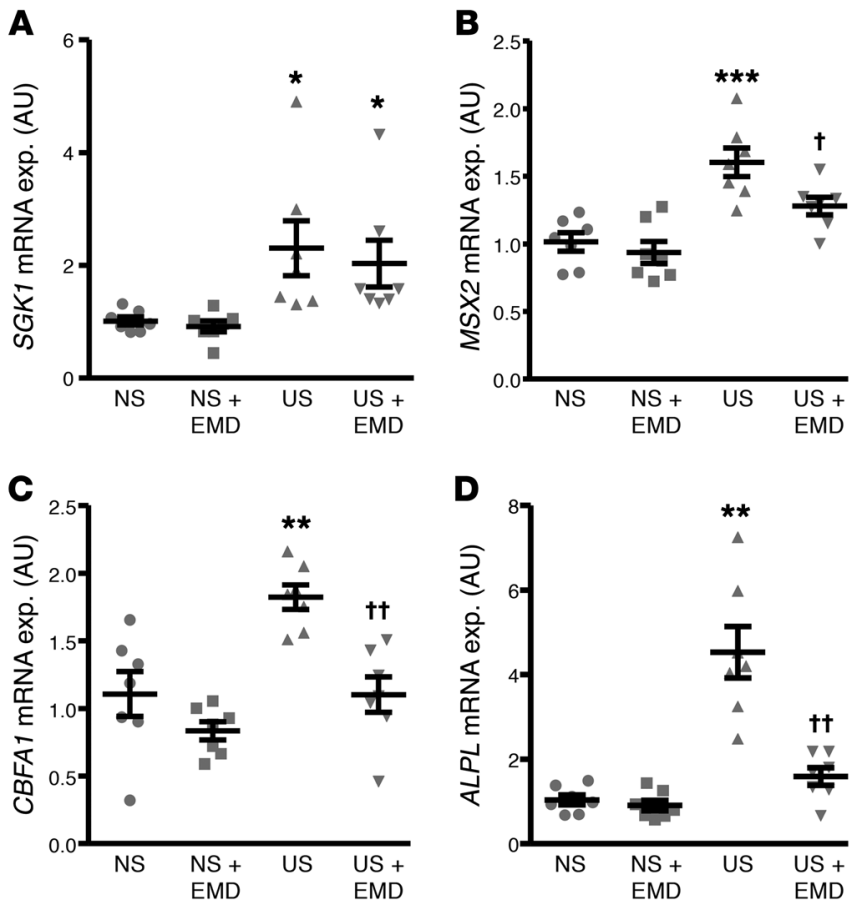

$\mathrm{apoE}^{-/-} \mathrm{sgk1}^{-/-}$mice, a difference, however, not reaching statistical significance. Similarly, plasma FGF23 C-term concentrations were significantly increased after subtotal nephrectomy in both genotypes, but tended to be lower in apoE ${ }^{-/-} \mathrm{sgk} 1^{-/-}$mice than in apoE $\mathrm{E}^{-/-} \mathrm{sgk} 1^{+/+}$mice, a difference not reaching statistical significance (Supplemental Table 4). Plasma cholesterol, aldosterone, and cortisol levels significantly increased following subtotal nephrectomy in apoE $\mathrm{E}^{-/-} \mathrm{sgk} 1^{+/+}$mice, but not in apoE $\mathrm{E}^{-/-} \mathrm{sgk1} 1^{-/-}$mice (Supplemental Table 4).

In apoE $E^{-/-} \mathrm{sgk1}^{+/+}$mice, the aortic Sgk1 mRNA expression was significantly upregulated by subtotal nephrectomy (Figure 9A). Furthermore, subtotal nephrectomy slightly but significantly increased the aortic arch calcium content (Figure 9B) without visible calcifications in von Kossa staining of thoracic aorta sections (Supplemental Figure 7). Furthermore, subtotal nephrectomy significantly upregulated the mRNA expression of stiffness markers Col1a1 and $F n 1$ in the vascular tissue (Supplemental Figure 8) and of hypertrophy markers Nppa and Nppb in the cardiac tissue (Supplemental Figure 9) from apoE $\mathrm{E}^{-/-} \mathrm{sgk1} 1^{+/+}$mice, all effects ameliorated in the apoE $\mathrm{E}^{-/-} \mathrm{sgk} 1^{-/-}$mice. Moreover, subtotal nephrectomy significantly increased Msx 2 mRNA and protein expression as well as Wnt7a, Wnt3a, Cbfa1, and Alpl mRNA levels in the aortic tissue of apoE $\mathrm{E}^{-/}$ sgk1 $1^{+/+}$mice (Figure 9, C-H). Similarly, the aortic mRNA expression of $Z f p 36$ was significantly higher and that of Ankh significantly lower after subtotal nephrectomy in apoE $\mathrm{E}^{-/-} \mathrm{sgk}^{+/+}$mice than in control mice (Figure 9, I and J). All effects on aortic osteoinductive signaling were significantly blunted in the apoE $\mathrm{E}^{-/-} \mathrm{sgk1}^{-/-}$mice (Figure 9, C-J). Thus, Sgk1 deficiency tends to improve renal function and protects against vascular calcification following subtotal nephrectomy.

Effect of SGK1 inhibition in primary HAoSMCs during uremic conditions. Further experiments were conducted to investigate the effects of SGK1 inhibition on osteo-/chondrogenic transdifferentiation of HAoSMCs during uremic conditions. Serum was collected
Figure 10. SGK1 inhibition ameliorates uremic serum-induced osteo-/ chondrogenic transdifferentiation of primary HAoSMCs. Scatter dot plots and arithmetic means \pm SEM ( $n=7$ per group; AU) of SGK1 (A), MSX2 (B), CBFA1 (C), and ALPL (D) relative mRNA expression in HAoSMCs following treatment with normal serum (NS) or uremic serum (US) without or with additional treatment with SCK1 inhibitor EMD638683 (EMD). ${ }^{*} P<0.05$, ${ }^{* *} P<0.01,{ }^{* *} P<0.001$ statistically significant vs. NS-treated HAoSMCs; ${ }^{\dagger} P<0.05,{ }^{\dagger} P<0.01$ statistically significant vs. US-treated HAoSMCs (Steel-Dwass method for $\mathbf{A}$ and 1-way ANOVA with Tukey-HSD post hoc test for $\mathbf{B}$ and $\mathbf{C}$ or with Games-Howell post hoc test for $\mathbf{D})$.

from hemodialysis patients before dialysis (30) and from healthy volunteers and HAoSMCs were exposed to uremic serum (US) or normal serum (NS) (Supplemental Table 5). Serum calcification propensity (30) measured as calciprotein particle maturation time $\left(T_{50}\right)$ was significantly higher in uremic serum than in normal serum (Supplemental Table 5). As shown in Figure 10A, treatment with uremic serum significantly upregulated SGK1 mRNA expression in HAoSMCs as compared with normal serum-treated HAoSMCs. Similarly, MSX2, CBFA1, and ALPL mRNA expression was significantly upregulated in uremic serum-treated HAoSMCs as compared with normal serum-treated HAoSMCs, effects significantly reduced by additional treatment with the SGK1 inhibitor EMD638683 (Figure 10, B-D). SGK1 inhibition, thus, interferes with osteo-/chondrogenic signaling in HAoSMCs following exposure to uremic serum.

\section{Discussion}

The current observations identify SGK1 as a decisive regulator of vascular calcification. SGK1 induces osteo-/chondrogenic reprogramming of VSMCs, at least partly, via NF- $\kappa \mathrm{B}$ activation, while SGK1 inhibition blunts or virtually abrogates vascular calcification in vitro and in vivo.

$S G K 1$ gene expression is highly dynamic, and strong upregulation of $S G K 1$ is observed in the cardiovascular system under several pathophysiological conditions $(16,21)$. SGK1 gene expression is regulated by various clinically important factors contributing to vascular calcification $(15,31)$. Accordingly, we demonstrated that SGK1 mRNA and protein expression in VSMCs was upregulated by dexamethasone and aldosterone as well as phosphate exposure. Phosphate exposure triggers upregulation of TGF- $\beta$ and BMP-2, which augment the osteo-/chondrogenic transdifferentiation of VSMCs $(32,33)$. Treatment with either TGF- $\beta$ or BMP- 2 increases SGK1 expression. Interestingly, high extracellular glucose concentrations also upregulated SGK1 expression in VSMCs. Like patients with CKD, diabetic patients develop vascular calcification (34). Increased aortic $S g k 1$ mRNA expression was also observed in 2 mouse models of CKD-related vascular calcification, klothohypomorphic mice and subtotal nephrectomy-treated mice. We also observed increased mRNA expression of SGK1 in coronary arteries of patients with impaired renal function (31). In end-stage CKD patients, SGK1 protein abundance was increased and localized at the calcified regions in coronary arteries. Along those lines, treatment of VSMCs with uremic serum increased SGK1 mRNA expression. Taken together, these observations suggest that SGK1 is dynamically upregulated in the vasculature under calcifying conditions, and is required for the triggering of vascular calcification. 
SGK1 is a powerful regulator of NF- $\kappa B$ activity (16). SGK1 stimulates IKK $\alpha$ with subsequent activation of $N F-\kappa B$, an effect requiring IKK $\beta$ (27). Accordingly, silencing or pharmacological inhibition of either IKK $\alpha$ or IKK $\beta$ abrogates SGK1-dependent signaling. The transcription factor NF- $\mathrm{KB}$ is essential for the development of vascular calcification and is activated in patients with CKD (28). Sustained activation of NF- $\kappa \mathrm{B}$ has been described following phosphate exposure of VSMCs (35). NF- $\kappa \mathrm{B}$ upregulates the osteogenic transcription factor MSX2, which is required for ALPL expression (36). Furthermore, vascular NF- $\kappa \mathrm{B}$ promotes expression of CBFA1, which also increases ALPL expression and is required for phosphate-induced vascular calcification (37-39). ALPL degrades $\mathrm{PPi}$, an inhibitor of hydroxyapatite formation and mineral deposition in the vascular tissue (8). ALPL overexpression is sufficient to cause generalized vascular calcification and has emerged as a key enzyme in vascular calcification (40). A downstream product of NF- $\kappa \mathrm{B}$ activation is TTP (41), an RNA-destabilizing factor that inhibits ANKH expression and, thus, the export of PPi in the extracellular space $(8,28)$. SGK1-induced decrease in PPi levels may result from both enhanced ALPL activity and reduced ANKH expression. However, SGK1 regulates a wide variety of further cellular functions and pathways (16). Other signaling pathways contributing to the effects of SGK1 on vascular calcification and VSMC osteo-/chondrogenic transdifferentiation, thus, cannot be ruled out. Nevertheless, NF-кB interference disrupts the osteoinductive effects during overexpression of constitutively active SGK1. NF-кB activation seems, therefore, to be required for the SGK1-dependent osteo-/chondrogenic transdifferentiation of VSMCs.

Along these lines, SGK1 regulates inflammatory processes in macrophages, at least partly, by NF- $\mathrm{BB}$ activation (22). SGK1 also fosters migration of VSMCs (23). SGK1 has therefore been associated with intimal atherosclerosis and vascular inflammation (22). Other than intimal atherosclerosis, phosphate-induced medial vascular calcification is mediated mainly by osteo-/chondrogenic transdifferentiation of VSMCs (34). Although overlapping pathways exist, intimal atherosclerosis and medial vascular calcification have been considered as distinct entities (42). The current observations indicate that SGK1 activation may be decisive for vascular calcification during CKD or further hyperphosphatemic conditions. Sgk1 deficiency and Sgk1 inhibition blunt vascular calcification and vascular stiffness in the cholecalciferol overload model. Calcitriol levels are decreased in patients with CKD (43). The cholecalciferol overload model, nonetheless, mimics vascular calcification and osteo-/ chondrogenic remodeling (44). Furthermore, the subtotal nephrectomy model exhibited vascular osteo-/chondrogenic reprogramming and slightly increased calcification, indicative of early-stage calcification. Sgk1 deficiency ameliorated vascular osteoinduction and calcification. However, Sgk1 deficiency also tended to improve renal function and to partially reverse hyperphosphatemia, dyslipidemia, hyperaldosteronism, and hypercortisolism in these mice. Thus, additional mechanisms may contribute to the beneficial vascular effects of Sgk1 deficiency. Also, beneficial effects of Sgk1 deficiency on other organs besides the vasculature may contribute to the observed phenotype. In any case, the results observed in the subtotal nephrectomy model indicate that SGK1 is dispensable during renal failure and overall suggest beneficial effects of SGK1 blockade as a therapeutic target in renal disease.
SGK1 has previously been suggested as a therapeutic target in cardiovascular disease, and a specific inhibitor that has already been tested in vivo is available $(16,26)$. Treatment with the SGK1 inhibitor EMD638683 strongly blunted vascular osteoinduction, stiffening, and calcification in the cholecalciferol overload model. SGK1 inhibition also ameliorates the osteoinductive properties of uremic serum, underscoring the feasibility of SGK1 inhibition in CKD patients. SGK1 is apparently not required for basic cellular functions, and exerts effects especially under pathophysiological conditions (45, 46). SGK1 is upregulated by phosphate exposure, glucocorticoids, aldosterone, and enhanced glucose concentrations, indicating that SGK1 could be a converging key signaling element mediating the effects of various procalcific signals. Accordingly, SGK1 inhibition also reduces aldosterone-induced VSMC osteoinduction.

Vascular calcification is observed not only in CKD, but also in further clinical conditions and in the aging common population (47). It is tempting to speculate that SGK1 similarly influences vascular calcification in those conditions and during vascular aging. As a matter of fact, mineralocorticoid receptor-sensitive upregulation of SGK1 expression has been shown to accelerate aging of the skin (48). SGK1 inhibition would further be expected to interfere with organ fibrosis, sodium reabsorption, metabolic syndrome, and thrombocyte aggregation as well as cardiac hypertrophy and failure $(16,21,22,49)$. SGK1 inhibition may therefore prove a clinically effective treatment strategy and warrants further study of the translational potential.

In conclusion, SGK1 is upregulated in calcifying conditions. SGK1 promotes vascular calcification, at least in part, by activating the NF- $\kappa \mathrm{B}$ pathway. SGK1 inhibition ameliorates vascular calcification in vitro and in vivo. These observations warrant further study of SGK1 inhibition as a therapeutic strategy in renal disease.

\section{Methods}

Cell culture of HAoSMCs. Primary human aortic smooth muscle cells (HAoSMCs) were commercially obtained from Thermo Fisher Scientific. HAoSMCs were routinely cultured in medium containing a 1:1 ratio of Waymouth's MB 752/1 medium and Ham's F-12 nutrient mixture (Thermo Fisher Scientific) supplemented with 10\% FBS (Thermo Fisher Scientific), $100 \mathrm{U} / \mathrm{ml}$ penicillin, and $100 \mu \mathrm{g} / \mathrm{ml}$ streptomycin (Thermo Fisher Scientific). HAoSMCs were grown to confluence and used in all experiments from passages 4 to 10. At least 8 different batches of HAoSMCs were used during the course of this study, and each experiment was performed in at least 2 different batches of HAoSMCs depending on the availability of the cells ( $n$ indicates the number of independent experiments performed at different passages of the cells). The medium was changed to medium containing $10 \%$ charcoal-stripped FBS (Sigma-Aldrich) 24 hours before aldosterone/ vehicle treatments to reduce the effects of endogenous ligands (13).

HAoSMCs were transfected with $2 \mu \mathrm{g}$ DNA encoding constitutively active $S G K 1^{\mathrm{S} 422 \mathrm{D}}$, inactive $\mathrm{SGK} 1^{\mathrm{K} 127 \mathrm{~N}}$ in $\mathrm{pcDNA} 3.1$ vector, or empty vector as control (18) using X-tremeGENE HP DNA transfection reagent (Roche Applied Science) according to the manufacturer's protocol. The cells were used 24 hours (confocal microscopy), 48 hours (RT-qPCR, luciferase assay, Western blotting), or 7 days (ALPL activity) after transfection. HAoSMCs were transfected with 10 nM SGK1 siRNA (s740, Thermo Fisher Scientific), 10 nM IKK $\alpha$ siRNA (s3076, Thermo Fisher Scientific), or 10 nM IKK $\beta$ siRNA (s223928, Thermo 
Fisher Scientific) or with $10 \mathrm{nM}$ negative control siRNA (4390843, Thermo Fisher Scientific) using siPORT amine transfection agent (Thermo Fisher Scientific) according to the manufacturer's protocol. The cells were used 48 hours (RT-qPCR), 7 days (ALPL activity), or 14 days (calcium deposition) after silencing. Transfection and silencing efficiency were determined by real-time quantitative PCR (RT-qPCR).

HAoSMCs were treated for 24 hours (RT-qPCR, confocal microscopy, Western blotting) (35), 7 days (ALPL activity), or 14 days (calcium deposition) with $2 \mathrm{mM} \beta$-glycerophosphate (Sigma-Aldrich), $50 \mu \mathrm{M}$ SGK1 inhibitor EMD638683 (stock in DMSO) (18), $10 \mu \mathrm{M}$ BAY11-7082 (stock in DMSO; Sigma-Aldrich), $10 \mu$ M BMS-345541 (stock in DMSO; Sigma-Aldrich), $10 \mu \mathrm{M}$ parthenolide (stock in DMSO; Sigma-Aldrich), $100 \mathrm{nM}$ aldosterone (stock in DMSO; Sigma-Aldrich), $10 \mu \mathrm{M}$ spironolactone (stock in DMSO; Sigma-Aldrich), $100 \mathrm{nM}$ dexamethasone (stock in ethanol; Sigma-Aldrich), $25 \mathrm{mM}$ D-glucose (Sigma-Aldrich), $10 \mathrm{ng} / \mathrm{ml}$ TGF- $\beta 1$ (stock in $4 \mathrm{mM} \mathrm{HCl}$ solution containing $1 \mathrm{mg} / \mathrm{ml} \mathrm{BSA}$; R\&D Systems), or $200 \mathrm{ng} / \mathrm{ml} \mathrm{BMP-2} \mathrm{(stock} \mathrm{in} 4 \mathrm{mM} \mathrm{HCl}$ solution; R\&D Systems). Equal amounts of vehicle were used as control. Treatment for 14 days with $3 \mathrm{mM}$ sodium phosphate buffer (Sigma-Aldrich) or with $10 \mathrm{mM} \beta$-glycerophosphate and $1.5 \mathrm{mM} \mathrm{CaCl}_{2}$ (Sigma-Aldrich) was used as calcification media for the calcium deposition quantification or for alizarin red staining, respectively. Fresh media with agents were added every 2-3 days.

Isolation and culture of primary MAoSMCs. Primary mouse aortic smooth muscle cells (MAoSMCs) were isolated from Sgk1-deficient and corresponding WT mice. The origin of the mice has been described previously (46). MAoSMCs were obtained from pooled aortic tissue similarly to what has been previously described (31). MAoSMCs were grown to confluence in DMEM/F12 medium (Thermo Fisher Scientific) supplemented with 10\% FBS (Thermo Fisher Scientific), $100 \mathrm{U} /$ $\mathrm{ml}$ penicillin, $100 \mu \mathrm{g} / \mathrm{ml}$ streptomycin (Thermo Fisher Scientific), and $0.025 \mu \mathrm{g} / \mathrm{ml}$ Fungizone (Thermo Fisher Scientific). The cells were used in all experiments from passages 3-6. $N$ indicates the number of independent experiments from 3 independent isolations of MAoSMCs. The cells were treated for 24 hours (RT-qPCR, confocal microscopy, luciferase assay) or 7 days (ALPL activity) with $2 \mathrm{mM} \beta$-glycerophosphate (Sigma-Aldrich). Treatment for 14 days with $3 \mathrm{mM}$ sodium phosphate buffer (Sigma-Aldrich) or with $10 \mathrm{mM} \beta$-glycerophosphate and $1.5 \mathrm{mM} \mathrm{CaCl}_{2}$ (Sigma-Aldrich) was used as calcification media for the calcium deposition quantification or for alizarin red staining, respectively. Fresh media with agents were added every 2-3 days.

Animal experiments. Sgk1-deficient and corresponding WT mice were injected s.c. with $400,000 \mathrm{IU} / \mathrm{kg}$ body weight of cholecalciferol (Sigma-Aldrich) or vehicle for 3 days as previously described $(6,44)$. For EMD638683 experiments, C57BL/6 mice received twice-daily oral gavage with $300 \mathrm{mg} / \mathrm{kg}$ body weight EMD638683 (Biorbyt); control mice received only vehicle. The mice were injected s.c. with 400,000 $\mathrm{IU} / \mathrm{kg}$ body weight of cholecalciferol (Sigma-Aldrich) or vehicle for 3 days. Pulse propagation velocity was measured in the abdominal aorta during isoflurane anesthesia (1\%-1.5\%) by ultrasound (Vevo 3100 , MX700 transducer) using EKV-image acquisition. Analysis was performed with the Vevo-Vasc software (Visualsonics, Fujifilm). After 6 days of treatment, mice were sacrificed in isoflurane anesthesia, blood was collected, and tissues were snap-frozen in liquid nitrogen.

Klotho-hypomorphic $(k l / k l)$ mice and corresponding WT mice were described earlier $(50,51)$. Mice were sacrificed and aortic tissue stored in liquid nitrogen.
Subtotal nephrectomy was performed in apolipoprotein E-deficient mice with or without Sgk1 deficiency. The origin of these mice was described previously (22). Subtotal nephrectomy was performed during isoflurane anesthesia (1.5\%-2\%) as previously described (6). In a first step, the right kidney mass was reduced by electrocauterization and surgical excision. In a second step, the left kidney was excised. Sham-operated mice were used as control. Buprenorphine $(0.05 \mathrm{mg} / \mathrm{kg}$ body weight s.c.) was used for analgesia after the surgical treatments. One week after the nephrectomy, BUN was measured and the diet changed to a phosphate-rich diet $(0.6 \%$ calcium and $0.9 \%$ phosphorous; C1031 mod, Altromin). After 6 weeks of treatment, mice were sacrificed, and blood was collected and tissues stored in liquid nitrogen. The subtotal nephrectomy procedure (6) was also performed in DBA mice and tissues collected after 16 weeks of treatment.

Plasma concentrations of phosphate, calcium, BUN, and total cholesterol were measured by a photometric method (FUJI FDC 3500i, Sysmex). For EMD638683 experiments, plasma concentrations of phosphate and calcium were measured by QuantiChrom Phosphate assay kit and QuantiChrom Calcium assay kit, respectively (BioAssay Systems). ELISA kits were used to determine plasma aldosterone (Alpha Diagnostic International), cortisol (Alpha Diagnostic International), and FGF23 C-term (Immutopics) concentrations according to the manufacturer's instructions.

Aortic stiffness measurements ex vivo. The abdominal aortae were isolated from mice under inhalation with isoflurane anesthesia and sacrificed by cervical dislocation. Aortae were quickly transferred to cold $\left(4^{\circ} \mathrm{C}\right)$, oxygenated $\left(95 \% \mathrm{O}_{2} / 5 \% \mathrm{CO}_{2}\right)$ physiological salt solution (PSS) containing (in mmol/l) $119 \mathrm{NaCl}, 4.7 \mathrm{KCl}, 1.2 \mathrm{KH}_{2} \mathrm{PO}_{4}, 25$ $\mathrm{NaHCO}_{3}, 1.2 \mathrm{MgSO}_{4}, 11.1$ glucose, $1.6 \mathrm{CaCl}_{2}$. After cleaning of the connective tissue with scissors without damaging the adventitia, the aortic ring segments were dissected into $2-\mathrm{mm}$ (vessel length) rings. Each ring was positioned between 2 stainless steel wires (diameter $0.0394 \mathrm{~mm}$ ) in a 5-ml organ bath of a Small Vessel Myograph (DMT 610M, Danish Myo Technology). The organ bath was filled with PSS. The bath solution was continuously oxygenated $\left(95 \% \mathrm{O}_{2} / 5 \% \mathrm{CO}_{2}\right)$ and kept at $37^{\circ} \mathrm{C}(\mathrm{pH} 7.4)(52)$. The vessels were allowed to equilibrate for 30 minutes in the absence of tension. For length/tension analysis, aortae were stretched to an internal circumference (10) that reproduces the wall force exerted on the vessel at a resting transmural pressure of $100 \mathrm{mmHg}$ (DMT Normalization module by CHART software). Thereafter, wall tension was measured by sequential increasing of the distance (stretch length) between the wires in $50-\mu \mathrm{m}$ increments using the software Chart5 (AD Instruments Ltd.). Wall tension was measured for 2 minutes per aorta after the stretch length was increased (52).

Calcification analysis. The quantification of aortic arch calcification was performed by incubation of the tissues overnight at $37^{\circ} \mathrm{C}$ in $0.6 \mathrm{M}$ $\mathrm{HCl}$. The calcium content in the supernatant was determined with a QuantiChrom Calcium assay kit (BioAssay Systems) according to the manufacturer's protocol. Tissues were lysed with $0.1 \mathrm{M} \mathrm{NaOH} / 0.1 \%$ SDS, and total protein concentration was measured by the Bradford assay (Bio-Rad Laboratories). VSMCs were decalcified for 24 hours at $4^{\circ} \mathrm{C}$ in $0.6 \mathrm{M} \mathrm{HCl}$. Calcium content was determined with a QuantiChrom Calcium assay kit (BioAssay Systems). VSMCs were lysed with $0.1 \mathrm{M} \mathrm{NaOH} / 0.1 \%$ SDS, and total protein concentration was measured by the Bradford assay (Bio-Rad Laboratories). Calcium content was normalized to total protein concentration. 
To visualize calcium deposition, aortae were stained with alizarin red $(0.0016 \%$ in $0.5 \% \mathrm{KOH}$; Sigma-Aldrich). VSMCs were fixed with $4 \%$ paraformaldehyde and stained with $2 \%$ alizarin red ( $\mathrm{pH} 4.5)$. Calcified areas are shown as red staining.

Paraformaldehyde-fixed thoracic aortic tissues were cryoprotected in $30 \%$ sucrose, frozen in mounting medium (Tissue-Tek, Sakura Finetek), and sectioned at a thickness of $8 \mu \mathrm{m}$ on coated slides. Sections were stained for calcification with a von Kossa staining kit (Abcam) according to the manufacturer's protocol. Calcified areas are shown as gray/black staining.

Alkaline phosphatase (ALPL) activity assay. ALPL activity in VSMCs was determined with an ALPL colorimetric assay kit (Abcam) according to the manufacturer's protocol. ALPL activity was normalized to total protein concentration as assessed by the Bradford assay (Bio-Rad Laboratories).

Luciferase assay. VSMCs were transfected for 48 hours with $1 \mu \mathrm{g}$ DNA mixture of NF- $\kappa \mathrm{B}-$ responsive luciferase construct and a constitutively expressing Renilla construct (40:1 ratio; Qiagen) as control for transfection efficiency using X-tremeGENE HP DNA transfection reagent (Roche Applied Science) according to the manufacturer's protocol. After the incubation period, cells were lysed with Passive Lysis Buffer (Promega) and assayed for transcriptional activity using Dual-Luciferase Reporter Assay (Promega) and a luminometer (Walter Wallac 2 plate reader, Perkin Elmer) according to the manufacturer's protocol. All results are expressed as the ratio of NF- $\kappa \mathrm{B}$ firefly luciferase to Renilla luciferase (relative light units) normalized to vector-transfected HAoSMCs or control-treated sgk $1^{+/+}$MAoSMCs, respectively.

CBFA1 transcription factor assay. The preparation of nuclear extracts from HAoSMCs was performed using NE-PER nuclear and cytoplasmic extraction reagents (Thermo Fisher Scientific) according to the manufacturer's instructions. Protein concentration was determined by Bradford assay (Bio-Rad Laboratories). Equal amounts of nuclear proteins were used to determine CBFA1 transcriptional activation with a RUNX2 Transcription Factor Colorimetric Assay Kit (Abcam) according to the manufacturer's protocol. Results are shown normalized to the control group.

PPi levels in cell culture medium. Medium PPi levels were determined with a PiPer Pyrophosphate assay kit (Thermo Fisher Scientific) according to the manufacturer's instructions. For each sample, the blank (reaction without pyrophosphatase) was subtracted to obtain the true PPi amount. PPi levels were normalized to total protein concentration as assessed by the Bradford assay (Bio-Rad Laboratories) and to the control group.

Quantitative RT-PCR. Total RNA was isolated from VSMCs and mouse tissues with Trifast Reagent (Peqlab) according to the manufacturer's instructions. Reverse transcription of total RNA was performed using oligo- $\mathrm{dT}_{12-18}$ primers (Thermo Fisher Scientific) and SuperScript III Reverse Transcriptase (Thermo Fisher Scientific). RT-qPCR was performed with the iCycler iQ Real-Time PCR Detection System (BioRad Laboratories) and iQ SYBR Green Supermix (Bio-Rad Laboratories) according to the manufacturer's instructions. A detailed description of the primer sequences (Thermo Fisher Scientific) can be found in Supplemental Methods online. The specificity of the PCR products was confirmed by analysis of the melting curves. All PCRs were performed in duplicate, and relative mRNA fold changes were calculated by the $2^{-\Delta \Delta \mathrm{Ct}}$ method using GAPDH as internal reference.

Western blot analysis. HAoSMCs were lysed with ice-cold IP lysis buffer (Thermo Fisher Scientific) supplemented with complete protease and phosphatase inhibitor cocktail (Thermo Fisher Scientific).
After centrifugation at 10,261 $g$ for 5 minutes, the proteins were boiled in Roti-Load1 Buffer (Carl Roth $\mathrm{GmbH}$ ) at $100^{\circ} \mathrm{C}$ for 10 minutes. Equal amounts of proteins were separated on SDS-polyacrylamide gels and transferred to PVDF membranes. The membranes were incubated with the following primary antibodies overnight at $4^{\circ} \mathrm{C}$ : rabbit anti-SGK1 (diluted 1:1,000; no. 12103, Cell Signaling), rabbit antiphospho-NDRG1 ( $\mathrm{Thr}^{346}$ ) (diluted 1:1,000; no. 3217, Cell Signaling), or rabbit anti-GAPDH (diluted 1:5,000; no. 2118, Cell Signaling); and then with secondary anti-rabbit HRP-conjugated antibody (diluted 1:1,000; Cell Signaling) for 1 hour at room temperature. For loading controls, the membranes were stripped in stripping buffer (Thermo Fisher Scientific) at room temperature for 10 minutes. Antibody binding was detected with ECL detection reagent (Thermo Fisher Scientific). Bands were quantified with ImageJ software (NIH), and results are shown as the ratio of total protein to GAPDH and phosphorylated protein to GAPDH normalized to the control groups.

Immunocytochemistry and confocal microscopy. VSMCs cultured onto 4-well chamber slides (BD Biostatus) were fixed with ice-cold $100 \%$ methanol for 10 minutes at room temperature (for NF-кB p65 staining) or with $4 \%$ paraformaldehyde/PBS for 15 minutes at room temperature and then permeabilized with PBS/0.1\% Triton X for 10 minutes at room temperature (for Sgk1 staining). Slides were incubated with $5 \%$ normal goat serum in PBS/0.1\% Triton X-100 for 1 hour at room temperature to reduce nonspecific background staining. Cells were incubated overnight at $4^{\circ} \mathrm{C}$ with primary rabbit polyclonal anti-NF-кB p65 antibody (diluted 1:50; sc-372, Santa Cruz Biotechnology) or with rabbit anti-mouse Sgk1 antibody (diluted 1:50; Pineda; ref. 18) and then with goat anti-rabbit Alexa Fluor 488conjugated antibody (diluted 1:1,000; Thermo Fisher Scientific) for 1 hour at room temperature. Nuclei were stained using DRAQ5 dye (diluted 1:1,000; Biostatus) for 10 minutes at room temperature. The slides were mounted with Prolong Gold antifade reagent (Thermo Fisher Scientific). Images were collected with a confocal laser-scanning microscope (LSM 510, Carl Zeiss MicroImaging GmbH) using a $\times 63$ (Watter), 1.2W objective. Negative controls were carried out simultaneously with all experiments by omitting incubation with primary antibody. The intensity of fluorescence per area was quantified with Image J software in at least 5 images taken in different areas within the sample, and the results are shown as arbitrary units normalized to the control group.

Immunohistochemistry and confocal microscopy. Murine thoracic aortic tissues were fixed in $4 \%$ paraformaldehyde, cryoprotected in $30 \%$ sucrose, frozen in mounting medium (Tissue-Tek, Sakura Finetek), and sectioned at a thickness of $8 \mu \mathrm{m}$ on coated slides. For immunostaining, sections were dehydrated at room temperature for 30 minutes and fixed in $100 \%$ methanol for 10 minutes at room temperature. To reduce nonspecific background staining, slides were incubated with $5 \%$ normal goat serum or with $5 \%$ BSA in PBS/0.1\% Triton X-100 for 1 hour at room temperature. Sections were incubated overnight at $4^{\circ} \mathrm{C}$ with primary goat polyclonal anti-Msx2 antibody (diluted 1:50; sc-17729, Santa Cruz Biotechnology) or with rabbit anti-mouse Sgk1 antibody (diluted 1:50; Pineda). Binding of primary antibodies was visualized using goat anti-rabbit Alexa Fluor 488-conjugated antibody or donkey anti-goat Alexa Fluor 488conjugated antibody (diluted 1:1,000; Thermo Fisher Scientific) incubated for 1 hour at room temperature. Nuclei were stained using DRAQ5 dye (diluted 1:1,000; Biostatus) and actin using Rhodamine 
Phalloidin (diluted 1:100; Thermo Fisher Scientific). The slides were mounted with Prolong Gold antifade reagent (Thermo Fisher Scientific). Images were collected with a confocal imaging system (A1Rsi+, Nikon Instruments) using a $\times 40$ (Oil), 1.4NA objective. Negative controls were carried out simultaneously with all experiments by omitting incubation with primary antibodies. The intensity of fluorescence per area was quantified with ImageJ software in at least 2 sections of each animal and at least 5 images taken in different areas within the samples, and the results are shown as arbitrary units normalized to the control group.

Human samples. Human coronary arteries were obtained from brain-dead multi-organ donors. The origin and collection of the samples and the patient characteristics were described in detail previously (31).

Blood was collected from CKD patients before dialysis. Healthy volunteers served as controls. All patients and volunteers gave informed consent. Serum was obtained by immediate centrifugation and stored at $-80^{\circ} \mathrm{C}$. HAoSMCs were serum-starved for 24 hours prior to treatment for 24 hours with $15 \%$ uremic serum from hemodialysis patients (uremic serum) or control serum from matched healthy individuals (normal serum) collected as described previously (30). Serum calcification propensity was analyzed by determination of the one-half maximal transition time $\left(T_{50}\right)$ of in vitro transformation from primary to secondary calciprotein particles (30) as described by Pasch et al. (53) using a Nephelostar Plus nephelometer (BMG Labtech).

For histological analysis, coronary arteries of 5 patients with endstage CKD and calcified vessels and 5 control patients showing no vascular calcification were examined at the Department of Pathology, Universität Erlangen-Nürnberg. The inclusion criterion for control patients was an estimated glomerular filtration rate greater than 45 $\mathrm{ml} / \mathrm{min}$. In both groups, clinical information on preexisting coronary disease was not available. Laboratory chemistry was obtained from hospital records.

Immunohistochemical analysis and von Kossa staining in human samples. Paraffin-embedded arteries were deparaffinized and rehydrated, followed by antigen retrieval using a pressure cooker for 2.5 minutes in target retrieval solution (TRS; Dako $\mathrm{GmbH}$ ). After blocking of endogenous peroxidase with $3 \% \mathrm{H}_{2} \mathrm{O}_{2}$ and $1 \% \mathrm{BSA}$, sections were incubated overnight at $4^{\circ} \mathrm{C}$ with monoclonal rabbit anti-SGK1 (12103, Cell Signaling) diluted 1:100 in $50 \mathrm{mM}$ Tris (pH 7.5) supplemented with $0.05 \%$ Tween-20 (Tris buffer). After washing with Tris buffer, sections were incubated with biotinylated goat anti-rabbit IgG (Vector Laboratories). Bound secondary antibodies were detected using $A B C$ staining kit and DAB Immpact (both from Vector Laboratories). All sections were counterstained with hematoxylin and examined using light microscopy. As negative control for immunohistochemical staining, the primary antibody was replaced by nonimmune mouse or rabbit serum (BioGenex) or Tris buffer. Calcified arteries were detected with von Kossa staining using standard procedure.

Statistics. Data are shown as scatter dot plots and arithmetic mean \pm SEM. $N$ indicates the number of independent experiments performed at different passages of the cells or the number of mice or human patients examined, respectively. Normality was tested with the Shapiro-Wilk test. Non-normal data sets were transformed (log, reciprocal or sqrt) before statistical testing to provide normality according to the Shapiro-Wilk test. Statistical testing was performed by 1-way ANOVA followed by Tukey's test for homoscedastic data or the Games-Howell test for heteroscedastic data. Non-normal data were tested by the Steel-Dwass method. Two groups were compared by unpaired 2-tailed $t$ test or $U$ test. For correlation analysis, Pearson correlation test was performed. $P$ less than 0.05 was considered statistically significant.

Study approval. All animal experiments were conducted according to the recommendations of the Guide for the Care and Use of Laboratory Animals of the NIH (National Academies Press) as well as the German law for the welfare of animals, and reviewed and approved by the local government authority (Regierungspräsidium Tübingen, Germany; Lageso Berlin, Germany). Experiments with human tissues (31) and serum were reviewed and approved by the local ethics commission (Ethical Committee of the Charite, Berlin, Germany; Ethical Committee of the University of Erlangen-Nürnberg, Erlangen, Germany; Ethical Committee of the Medical University of Graz, Graz, Austria).

\section{Author contributions}

JV, FL, and IA designed research; JV, IA, TTDL, RT, KM, TA, XL, $\mathrm{CD}, \mathrm{DZ}, \mathrm{BB}$, and MS performed experiments; JV, BM, DK, MG, KA, DNM, BP, FL, and IA analyzed and interpreted data; JV, FL, and IA wrote the manuscript with comments and edits from all authors.

\section{Acknowledgments}

This work was supported by the Berlin Institute of Health Translational Postdoc Grant, Deutsche Forschungsgemeinschaft (AL2054/1-1, VO2259/2-1, GO766/12-3, GO766/22-1), the Sonnenfeld Foundation, the Else Kröner-Fresenius-Stiftung, and the European Union Seventh Framework Programme (FP7/2007-2013-603288-SysVasc). The authors gratefully acknowledge the technical assistance of A. Lange and the Advanced Medical Bioimaging Core Facility of the Charité for support in acquisition of the imaging data.

Address correspondence to: Jakob Voelkl, Department of Internal Medicine and Cardiology, Charité - Universitätsmedizin Berlin, Augustenburgerplatz 1, 13353 Berlin, Germany. Phone: 49.30.450.565074; Email: jakob.voelkl@charite.de.
1. Rennenberg RJ, Kessels AG, Schurgers LJ, van Engelshoven JM, de Leeuw PW, Kroon AA. Vascular calcifications as a marker of increased cardiovascular risk: a meta-analysis. Vasc Health Risk Manag. 2009;5(1):185-197.

2. Mizobuchi M, Towler D, Slatopolsky E. Vascular calcification: the killer of patients with chronic kidney disease. JAm Soc Nephrol. 2009;20(7):1453-1464.

3. Giachelli CM. The emerging role of phos- phate in vascular calcification. Kidney Int. 2009;75(9):890-897.

4. Chen NX, Moe SM. Pathophysiology of vascular calcification. Curr Osteoporos Rep. 2015;13(6):372-380.

5. Paloian NJ, Giachelli CM. A current understanding of vascular calcification in CKD. Am J Physiol Renal Physiol. 2014;307(8):F891-F900.

6. Alesutan I, et al. Augmentation of phosphateinduced osteo-/chondrogenic transformation of vascular smooth muscle cells by homoarginine. Cardiovasc Res. 2016;110(3):408-418.

7. Cheng SL, et al. Targeted reduction of vascular Msx1 and Msx2 mitigates arteriosclerotic calcification and aortic stiffness in LDLR-deficient mice fed diabetogenic diets. Diabetes. 2014;63(12):4326-4337.

8. Sheen CR, et al. Pathophysiological role of vascular smooth muscle alkaline phosphatase in medial artery calcification. J Bone Miner Res. 2015;30(5):824-836. 
9. Shroff RC, et al. Chronic mineral dysregulation promotes vascular smooth muscle cell adaptation and extracellular matrix calcification. J Am Soc Nephrol. 2010;21(1):103-112.

10. Hené RJ, Boer P, Koomans HA, Mees EJ. Plasma aldosterone concentrations in chronic renal disease. Kidney Int. 1982;21(1):98-101.

11. Matsumoto Y, et al. Spironolactone reduces cardiovascular and cerebrovascular morbidity and mortality in hemodialysis patients. J Am Coll Cardiol. 2014;63(6):528-536.

12. Tatsumoto N, et al. Spironolactone ameliorates arterial medial calcification in uremic rats: the role of mineralocorticoid receptor signaling in vascular calcification. Am J Physiol Renal Physiol. 2015;309(11):F967-F979.

13. Voelkl J, et al. Spironolactone ameliorates PIT1-dependent vascular osteoinduction in klotho-hypomorphic mice. J Clin Invest. 2013;123(2):812-822.

14. Gracia-Iguacel C, et al. Cortisol levels are associated with mortality risk in hemodialysis patients. Clin Nephrol. 2014;82(4):247-256.

15. Mori K, Shioi A, Jono S, Nishizawa Y, Morii H. Dexamethasone enhances In vitro vascular calcification by promoting osteoblastic differentiation of vascular smooth muscle cells. Arterioscler Thromb Vasc Biol. 1999;19(9):2112-2118.

16. Lang F, Voelkl J. Therapeutic potential of serum and glucocorticoid inducible kinase inhibition. Expert Opin Investig Drugs. 2013;22(6):701-714.

17. Bezzerides VJ, et al. Inhibition of serum and glucocorticoid regulated kinase-1 as novel therapy for cardiac arrhythmia disorders. Sci Rep. 2017;7(1):346

18. Voelkl J, et al. SGK1-sensitive regulation of cyclin-dependent kinase inhibitor 1B (p27) in cardiomyocyte hypertrophy. Cell Physiol Biochem. 2015;37(2):603-614

19. Voelkl J, et al. Sgk1 sensitivity of $\mathrm{Na}(+) / \mathrm{H}(+)$ exchanger activity and cardiac remodeling following pressure overload. Basic Res Cardiol. 2012;107(2):236

20. Voelk1 J, et al. Sgk1-dependent stimulation of car$\operatorname{diac} \mathrm{Na}^{+} / \mathrm{H}^{+}$exchanger Nhe1 by dexamethasone. Cell Physiol Biochem. 2013;32(1):25-38.

21. Lang F, Böhmer C, Palmada M, Seebohm G, Strutz-Seebohm N, Vallon V. (Patho)physiological significance of the serum- and glucocorticoid-inducible kinase isoforms. Physiol Rev. 2006;86(4):1151-1178.

22. Borst O, et al. Pivotal role of serum- and glucocorticoid-inducible kinase 1 in vascular inflammation and atherogenesis. Arterioscler Thromb Vasc Biol. 2015;35(3):547-557.

23. Walker-Allgaier B, et al. SGK1 up-regulates Orai1 expression and VSMC migration during neointima formation after arterial injury. Thromb Haemost. 2017;117(5):1002-1005.
24. Cheng J, Truong LD, Wu X, Kuhl D, Lang F, Du J. Serum- and glucocorticoid-regulated kinase 1 is upregulated following unilateral ureteral obstruction causing epithelial-mesenchymal transition. Kidney Int. 2010;78(7):668-678.

25. Voelkl J, et al. PKB/SGK-resistant GSK-3 signaling following unilateral ureteral obstruction. Kidney Blood Press Res. 2013;38(1):156-164.

26. Ackermann TF, Boini KM, Beier N, Scholz W, Fuchss T, Lang F. EMD638683, a novel SGK inhibitor with antihypertensive potency. Cell Physiol Biochem. 2011;28(1):137-146.

27. Tai DJ, Su CC, Ma YL, Lee EH. SGK1 phosphorylation of IkappaB kinase alpha and p300 up-regulates NF-kappaB activity and increases $\mathrm{N}$-methyl-D-aspartate receptor NR2A and NR2B expression. J Biol Chem. 2009;284(7):4073-4089.

28. Zhao G, et al. Activation of nuclear factor- $\mathrm{kB}$ accelerates vascular calcification by inhibiting ankylosis protein homolog expression. Kidney Int. 2012;82(1):34-44.

29. Quinkler M, et al. Increased expression of mineralocorticoid effector mechanisms in kidney biopsies of patients with heavy proteinuria. Circulation. 2005;112(10):1435-1443.

30. Voelkl J, et al. Zinc inhibits phosphate-induced vascular calcification through TNFAIP3mediated suppression of NF-kB. JAm Soc Nephrol. 2018;29(6):1636-1648.

31. Alesutan I, et al. Involvement of vascular aldosterone synthase in phosphate-induced osteogenic transformation of vascular smooth muscle cells. Sci Rep. 2017;7(1):2059.

32. Alesutan I, Musculus K, Castor T, Alzoubi K, Voelkl $\mathrm{J}$, Lang F. Inhibition of phosphate-induced vascular smooth muscle cell osteo-/chondrogenic signaling and calcification by bafilomycin A1 and methylamine. Kidney Blood Press Res. 2015;40(5):490-499.

33. Evrard S, Delanaye P, Kamel S, Cristol JP, Cavalier $\mathrm{E}, \mathrm{SFBC} / \mathrm{SN}$ joined working group on vascular calcifications. Vascular calcification: from pathophysiology to biomarkers. Clin Chim Acta. 2015;438:401-414.

34. Lanzer P, et al. Medial vascular calcification revisited: review and perspectives. Eur Heart J . 2014:35(23):1515-1525.

35. Zhao MM, et al. Mitochondrial reactive oxygen species promote $\mathrm{p} 65$ nuclear translocation mediating high-phosphate-induced vascular calcification in vitro and in vivo. Kidney Int 2011;79(10):1071-1079.

36. Lee HL, Woo KM, Ryoo HM, Baek JH. Tumor necrosis factor-alpha increases alkaline phosphatase expression in vascular smooth muscle cells via MSX2 induction. Biochem Biophys Res Commun. 2010;391(1):1087-1092.

37. Lin ME, Chen T, Leaf EM, Speer MY, Giachelli CM. Runx2 expression in smooth muscle cells is required for arterial medial calcification in mice.
Am J Pathol. 2015;185(7):1958-1969.

38. Raaz U, et al. Transcription factor Runx2 promotes aortic fibrosis and stiffness in type 2 diabetes mellitus. Circ Res. 2015;117(6):513-524.

39. Chen NX, et al. The mechanisms of uremic serum-induced expression of bone matrix proteins in bovine vascular smooth muscle cells. Kidney Int. 2006;70(6):1046-1053.

40. Savinov AY, Salehi M, Yadav MC, Radichev I, Millán JL, Savinova OV. Transgenic overexpression of tissue-nonspecific alkaline phosphatase (TNAP) in vascular endothelium results in generalized arterial calcification. JAm Heart Assoc. 2015;4(12):e002499.

41. King EM, Kaur M, Gong W, Rider CF, Holden NS, Newton R. Regulation of tristetraprolin expression by interleukin- $1 \beta$ and dexamethasone in human pulmonary epithelial cells: roles for nuclear factor-kappa B and p38 mitogenactivated protein kinase. J Pharmacol Exp Ther. 2009;330(2):575-585.

42. Amann K. Media calcification and intima calcification are distinct entities in chronic kidney disease. Clin J Am Soc Nephrol. 2008;3(6):1599-1605.

43. Lang F, Ritz E, Voelkl J, Alesutan I. Vascular calcification - is aldosterone a culprit? Nephrol Dial Transplant. 2013;28(5):1080-1084.

44. Alesutan I, et al. Inhibition of osteo/chondrogenic transformation of vascular smooth muscle cells by $\mathrm{MgCl} 2$ via calcium-sensing receptor. J Hypertens. 2017;35(3):523-532.

45. Lang F, Stournaras C, Alesutan I. Regulation of transport across cell membranes by the serumand glucocorticoid-inducible kinase SGK1. Mol Membr Biol. 2014;31(1):29-36.

46. Wulff $\mathrm{P}$, et al. Impaired renal $\mathrm{Na}(+)$ retention in the sgk1-knockout mouse. JClin Invest. 2002;110(9):1263-1268.

47. Marulanda J, Alqarni S, Murshed M. Mechanisms of vascular calcification and associated diseases. Curr Pharm Des. 2014;20(37):5801-5810.

48. Nagase T, et al. Aging-like skin changes in metabolic syndrome model mice are mediated by mineralocorticoid receptor signaling. Aging Cell. 2013;12(1):50-57.

49. Artunc F, Lang F. Mineralocorticoid and SGK1-sensitive inflammation and tissue fibrosis. Nephron Physiol. 2014;128(1-2):35-39.

50. Kuro-o M, et al. Mutation of the mouse klotho gene leads to a syndrome resembling ageing. Nature. 1997;390(6655):45-51.

51. Leibrock $\mathrm{CB}$, et al. $\mathrm{NH} 4 \mathrm{Cl}$ treatment prevents tissue calcification in Klotho deficiency. J Am Soc Nephrol. 2015;26(10):2423-2433.

52. Fésüs $\mathrm{G}$, et al. Adiponectin is a novel humoral vasodilator. Cardiovasc Res. 2007;75(4):719-727.

53. Pasch A, et al. Nanoparticle-based test measures overall propensity for calcification in serum. J Am Soc Nephrol. 2012;23(10):1744-1752. 